\title{
SHAPE DERIVATIVES FOR AN AUGMENTED LAGRANGIAN FORMULATION OF ELASTIC CONTACT PROBLEMS*
}

\author{
Bastien Chaudet-Dumas** and Jean Deteix
}

\begin{abstract}
This work deals with shape optimization of an elastic body in sliding contact (Signorini) with a rigid foundation. The mechanical problem is written under its augmented Lagrangian formulation, then solved using a classical iterative approach. For practical reasons we are interested in applying the optimization process with respect to an intermediate solution produced by the iterative method. Because of the projection operator involved at each iteration, the iterate solution is not classically shape differentiable. However, using an approach based on directional derivatives, we are able to prove that it is conically differentiable with respect to the shape, and express sufficient conditions for shape differentiability. Finally, from the analysis of the sequence of conical shape derivatives of the iterative process, conditions are established for the convergence to the conical derivative of the original contact problem.
\end{abstract}

Mathematics Subject Classification. 35J86, 49K40, 49Q10, 74M15, 74P15.

Received August 7, 2019. Accepted September 28, 2020.

\section{INTRODUCTION}

Structural optimization has become an integral part of industrial conception, with applications in more and more challenging mechanical contexts. Those contexts often lead to complex mathematical formulations involving non-linearities and/or non-differentiabilities, which causes many difficulties when considering the associated shape optimization or optimal control problem.

In this article, we study a shape optimization problem in the context of contact mechanics. Especially, the physical system models an elastic body (without restriction on its dimension) coming in sliding contact with a rigid foundation, which takes the mathematical form of an elliptic variational inequality (VI) of the first kind. As variational inequalities involve projection operators, differentiation with respect to the control parameter (in order to derive optimality conditions or use a gradient descent optimization method) is not an easy task.

Using the terminology from [15], let us gather the approaches to treat optimal control or shape optimization problems for variational inequalities in two families: the ones following the first optimize then discretize paradigm, and the others following the first discretize then optimize paradigm. In the first one, the idea is to work with a weaker notion of differentiability, namely conical differentiability (see Def. 2.3), in order to get

* This work was supported by the Natural Sciences and Engineering Research Council of Canada (NSERC).

Keywords and phrases: Shape and topology optimization, unilateral contact, elliptic variational inequalities, conical differentiability, augmented Lagrangian method.

Groupe Interdisciplinaire de Recherche en Éléments Finis de l'Université Laval, Départment de Mathématiques et Statistiques, Université Laval, Québec, Canada.

** Corresponding author: bastien.chaudet.1@ulaval.ca 
optimality conditions. Let us mention the pioneer work [24], as well as other works in the same direction [25], the series of works [28-35] and also [15, 19]. In those works, the conical derivative of the solution with respect to the control parameter is expressed by means of a variational inequality. Thus the optimality conditions obtained might be difficult to use in practice. However, in [27], for the two-dimensional Signorini problem, the authors give conditions for some specific functional to be shape differentiable. This allows them to get an explicit expression for the shape derivative of the functional using an adjoint state. In the second family of approaches, first the variational inequality is discretized, then tools from subdifferential calculus are used in the finite dimensional setting. The interested reader is referred to [11, 12, 21, 22] for shape optimization for the two-dimensional Signorini problem discretized using finite elements. The way the authors define the set of admissible shapes enable them to prove existence of an optimal design for the non-disctretized problem. Moreover, in [13], convergence analysis with respect to the discretization parameter is performed. In the same spirit, we finally mention the series of papers $[1,2,14]$ dedicated to shape optimization for the contact problem with Coulomb friction, which is much more cumbersome. There, the authors manage to characterize an outer approximation of the shape subdifferential of the functional to minimize, then use a bundle algorithm.

The approach proposed here follows the first optimize then discretize paradigm. The goal is to express first order optimality conditions for the shape optimization problem associated to some generic cost functional. It can somehow be understood as a continuation of [27]. In order to facilitate shape sensitivity of the contact problem, we write the sliding contact conditions using the normal to the rigid foundation instead of the normal to the body, as in [3]. Furthermore, the variational inequality arising from the basic formulation of sliding contact problems is transformed using an Augmented Lagrangian formulation (ALF) which we plan on solving using a basic iterative approach, the Augmented Lagrangian method (ALM). Aiming at practical use of the ALM in a numerical shape optimization process, we study consistency of this iterative process with respect to shape differentiation. In other words, we aim at deriving conditions for the shape derivatives of the iterates generated by this method to converge to the shape derivative of the solution to the original problem.

This work is structured as follows. Section 2 presents the original problem, its different formulations as well as some notations and notions related to convex analysis and conical differentiability. We also introduce the augmented Lagrangian method (ALM) applied to this problem. Section 3 is dedicated to shape optimization and is divided in three parts. In the first one, we give a proof (adapted from the classical one) that the solution of this formulation is conically differentiable. In the second one, we prove the same property for each of the iterates generated by the iterative algorithm. Convergence analysis of those conical shape derivatives to the one obtained for the original formulation is studied in the third part. Finally, we give the expression of the derivative of a general cost functional $J$ for a mechanical system in sliding contact with a rigid foundation.

\section{Problem Formulation}

\subsection{Geometrical setting}

Here, the body $\Omega \subset \mathbb{R}^{d}, d \in\{2,3\}$, is assumed to have $\mathcal{C}^{1}$ boundary, and to be in contact with a rigid foundation $\Omega_{\text {rig }}$, which has a $\mathcal{C}^{3}$ compact boundary $\partial \Omega_{\text {rig }}$, see Figure 1 . Let $\Gamma_{D}$ be the non-empty part of the boundary where a homogeneous Dirichlet condition applies (blue part), $\Gamma_{N}$ the part where a Neumann condition $\tau$ applies (orange part), $\Gamma_{C}$ the potential contact zone (green part), and $\Gamma$ the rest of the boundary, which is free of any constraint (i.e. homogeneous Neumann boundary condition). Those four parts are open, mutually disjoint and moreover: $\overline{\Gamma_{D}} \cup \overline{\Gamma_{N}} \cup \overline{\Gamma_{C}} \cup \bar{\Gamma}=\partial \Omega$. In order to avoid technical difficulties, it is assumed that $\overline{\Gamma_{C}} \cap \overline{\Gamma_{D}}=\emptyset$.

The outward normal to $\partial \Omega$ is denoted $\mathbf{n}_{\mathbf{o}}$. Similarly, the inward normal vector to $\partial \Omega_{\text {rig }}$ is denoted $\mathbf{n}$.

\subsection{Notation, function spaces and preliminaries}

Throughout this article, for any $\mathcal{O} \subset \mathbb{R}^{d}, L^{p}(\mathcal{O})$ represents the usual set of $p$ th power measurable functions on $\mathcal{O}$, and $\left(L^{p}(\mathcal{O})\right)^{d}=\mathbf{L}^{p}(\mathcal{O})$. The scalar product defined on $L^{2}(\mathcal{O})$ or $\mathbf{L}^{2}(\mathcal{O})$ is denoted (without distinction) by $(\cdot, \cdot)_{\mathcal{O}}$ and its norm $\|\cdot\|_{0, \mathcal{O}}$. 
The Sobolev spaces, denoted $W^{m, p}(\mathcal{O})$ with $p \in[1,+\infty], p$ integer are defined as

$$
W^{m, p}(\mathcal{O})=\left\{u \in L^{p}(\mathcal{O})\left|D^{\alpha} u \in L^{p}(\mathcal{O}) \forall\right| \alpha \mid \leq m\right\}
$$

where $\alpha$ is a multi-index in $\mathbb{N}^{d}$ and $\mathbf{W}^{m, p}(\mathcal{O})=\left(W^{m, p}(\mathcal{O})\right)^{d}$. The spaces $W^{s, 2}(\mathcal{O})$ and $\mathbf{W}^{s, 2}(\mathcal{O}), s \in \mathbb{R}$, are denoted $H^{s}(\mathcal{O})$ and $\mathbf{H}^{s}(\mathcal{O})$ respectively. Their norm are denoted $\|\cdot\|_{s, \mathcal{O}}$.

Without distinction for the dimension, we denote the duality pairing between $H^{\frac{1}{2}}(\mathcal{O})$ and its dual $H^{-\frac{1}{2}}(\mathcal{O})$ (or between $\mathbf{H}^{\frac{1}{2}}(\mathcal{O})$ and $\mathbf{H}^{-\frac{1}{2}}(\mathcal{O})$ ) by $\langle\cdot, \cdot\rangle_{\mathcal{O}}$. More generally, for a space $V$ and $V^{*}$ its dual, we denote the duality pairing by $\langle\cdot, \cdot\rangle_{V^{*}, V}$.

The subspaces of functions in $H^{s}(\mathcal{O})$ and $\mathbf{H}^{s}(\mathcal{O})$ that vanish on a part of the boundary $\Gamma \subset \partial \mathcal{O}$ are denoted $H_{\Gamma}^{s}(\mathcal{O})$ and $\mathbf{H}_{\Gamma}^{s}(\mathcal{O})$. In particular, we denote the vector space of admissible displacements $\mathbf{X}:=\mathbf{H}_{\Gamma_{D}}^{1}(\Omega)$.

The sets $\mathbb{T}^{2}$ and $\mathbb{T}^{4}$ are the sets of real valued tensors of order 2 and 4 respectively. For any $v$ vector in $\mathbb{R}^{d}$ or second order tensor in $\mathbb{T}^{2}$, the product with the normal $v \cdot \mathbf{n}_{\mathbf{o}}$ (respectively with the normal to the rigid foundation $v \cdot \mathbf{n}$ ) is denoted $v_{\mathbf{n}_{\mathbf{o}}}\left(\right.$ respectively $\left.v_{\mathbf{n}}\right)$. Similarly, the tangential part of $v$ is denoted $v_{\mathbf{t}_{\mathbf{o}}}=v-v_{\mathbf{n}_{\mathbf{o}}} \mathbf{n}_{\mathbf{o}}$ (respectively $\left.v_{\mathbf{t}}=v-v_{\mathbf{n}} \mathbf{n}\right)$.

Finally, we introduce some notations from convex analysis. The indicator function and the characteristic function of an arbitrary set $S$ are denoted $I_{S}$ and $\chi_{S}$, respectively, that is

$$
I_{S}(x):=\left\{\begin{array}{cc}
0 & \text { if } x \in S, \\
+\infty & \text { if } x \notin S,
\end{array} \chi_{S}(x):= \begin{cases}1 & \text { if } x \in S \\
0 & \text { if } x \notin S .\end{cases}\right.
$$

Let $H$ be a Hilbert space, and $C$ a non-empty closed convex subset of $H$, then for any $x \in H$, the unique projection of $x$ onto $C$ is denoted $\operatorname{Proj}_{C}(x)$. Moreover, if $b$ is a bilinear form inducing an inner product on $H$, the projection of $x$ onto $C$ with respect to this inner product is denoted $\operatorname{Proj}_{C}^{b}(x)$.

Let us now recall some notions related to conical differentiability, see [24].

If $H$ denotes a Hilbert space and $b$ a coercive symmetric bilinear form on $H \times H$, then for any $K \subset H$ and $y \in K$, we define:

- the polar cone of $K$ with respect to $b$ as $[K]_{b}^{0}:=\{x \in H: \forall z \in K, b(x, z) \leq 0\}$;

- the radial cone of $K$ at $y$ as $C_{y}(K):=\{w \in H: \exists t>0, y+t w \in K\}$;

- the tangent cone of $K$ at $y$ as $S_{y}(K):=\overline{C_{y}(K)}$;

- the cone $S^{y}(K):=S_{y}(K) \cap[\mathbb{R}(v-y)]_{b}^{0}$, where $v \in y+\left[S_{y}(K)\right]_{b}^{0}$ and $\mathbb{R}(v-y):=\{\eta(v-y) \mid \eta \in \mathbb{R}\}$.

Definition 2.1. Let $K \subset H$ be a closed convex set. $K$ is said to be polyhedric at $v \in H$ if, denoting $y=\operatorname{Proj}_{K}^{b}(v)$, one has:

$$
S^{y}(K)=\overline{C_{y}(K) \cap[\mathbb{R}(v-y)]_{b}^{0}} .
$$

Definition 2.2. Let $K \subset H$ be a closed convex set. $K$ is said to be polyhedric in $H$ if it is polyhedric at each $v \in H$.

Definition 2.3. Let $V_{1}, V_{2}$ be two Banach spaces. A continuous function $f: V_{1} \rightarrow V_{2}$ admits a conical derivative at $x$ if there exists an operator $Q: V_{1} \rightarrow V_{2}$ positively homogeneous such that:

$$
\forall h \in V_{1}, \forall t>0, \quad f(x+t h)=f(x)+t Q(h)+o(t) .
$$

Using these notations, we may recall one of the main results on the differentiability of projection operators, namely [24], Theorem 2.1. 


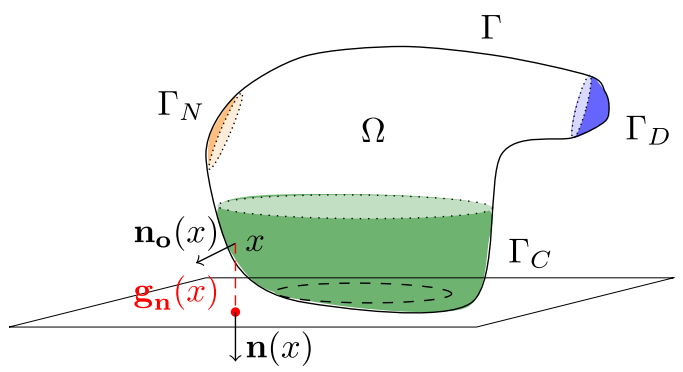

FIGURE 1. Elastic body in contact with a rigid foundation.

Theorem 2.4. Let $v \in H$ and $y=\operatorname{Proj}_{K}^{b}(v)$. If $K$ is polyhedric at $v$, then the projection $\operatorname{Proj}_{K}^{b}$ is conically differentiable at $v$, with conical derivative $\operatorname{Proj}_{S^{y}(K)}^{b}$. In other words, for all $w \in H$ and $t>0$ :

$$
\operatorname{Proj}_{K}^{b}(v+t w)=y+t \operatorname{Proj}_{S^{y}(K)}^{b}(w)+o(t) .
$$

\subsection{Mechanical model}

In this work the material is assumed to verify the linear elasticity hypothesis (small deformations and Hooke's law, see for example [4]), associated with the small displacements assumption (see [20]). The physical displacement is denoted $\mathbf{u}$, and belongs to $\mathbf{X}$. The stress tensor is defined by $\boldsymbol{\sigma}(\mathbf{u})=\mathbb{C}: \boldsymbol{\epsilon}(\mathbf{u})$, where $\boldsymbol{\epsilon}(\mathbf{u})=\frac{1}{2}\left(\nabla \mathbf{u}+\nabla \mathbf{u}^{T}\right)$ denotes the linearized strain tensor, and $\mathbb{C}$ is the elasticity tensor. This elasticity tensor is a fourth order tensor belonging to $L^{\infty}\left(\Omega, \mathbb{T}^{4}\right)$, and it is assumed to be elliptic (with constant $\alpha_{0}$ ). Regarding external forces, the body force $\mathbf{f} \in \mathbf{L}^{2}(\Omega)$, and traction (or surface load) $\boldsymbol{\tau} \in \mathbf{H}^{\frac{1}{2}}\left(\Gamma_{N}\right)$.

\subsection{Non-penetration condition}

At each point $x$ of $\Gamma_{C}$, let us define the gap $\mathbf{g}_{\mathbf{n}}(x)$, as the oriented distance function to the rigid foundation at $x$, see Figure 1 . Due to the regularity of the rigid foundation, there exists $h_{0}$ sufficiently small such that, for all $h<h_{0}$

$$
\partial \Omega_{r i g}^{h}:=\left\{x \in \mathbb{R}^{d}|| \mathbf{g}_{\mathbf{n}}(x) \mid<h\right\},
$$

is a neighborhood of $\partial \Omega_{\text {rig }}$ where $\mathbf{g}_{\mathbf{n}}$ is of class $\mathcal{C}^{3}$, see [5]. In particular, this ensures that $\mathbf{n}$ is well defined on $\partial \Omega_{\text {rig }}^{h}$, and that $\mathbf{n} \in \mathcal{C}^{2}\left(\partial \Omega_{\text {rig }}^{h}, \mathbb{R}^{d}\right)$. Moreover, in the context of small displacements, it can be assumed that the potential contact zone $\Gamma_{C}$ is such that $\Gamma_{C} \subset \partial \Omega_{\text {rig }}^{h}$. Hence there exists a neighborhood of $\Gamma_{C}$ such that $\mathbf{g}_{\mathbf{n}}$ and $\mathbf{n}$ are of class $\mathcal{C}^{3}$ and $\mathcal{C}^{2}$, respectively. Especially, this implies that the function $\mathbf{g}_{\mathbf{n}} \mathbf{n} \in \mathcal{C}^{2}\left(\partial \Omega_{\text {rig }}^{h}, \mathbb{R}^{d}\right)$. It can thus be extended to a function $\mathbf{g} \in \mathcal{C}^{2}\left(\mathbb{R}^{d}\right)$ such that $\mathbf{g}=0$ on $\mathbb{R}^{d} \backslash \partial \Omega_{\text {rig }}^{h^{\prime}}$ for some $h^{\prime}>h$. Since $\overline{\Gamma_{C}} \cap \overline{\Gamma_{D}}=\emptyset$, one has that $\overline{\Gamma_{D}} \subset\left(\mathbb{R}^{d} \backslash \partial \Omega_{r i g}^{h^{\prime}}\right)$ for $h, h^{\prime}$ small enough. Consequently, $\mathbf{g} \in \mathbf{X}$.

The non-penetration condition can be stated as follows: $\mathbf{u}_{\mathbf{n}} \leq \mathbf{g}_{\mathbf{n}}$ a.e. on $\Gamma_{C}$. Thus, we introduce the closed convex set of admissible deformations that realize this condition, see [8]:

$$
\mathbf{K}:=\left\{\mathbf{v} \in \mathbf{X} \mid \mathbf{v}_{\mathbf{n}} \leq \mathbf{g}_{\mathbf{n}} \text { a.e. on } \Gamma_{C}\right\}=\mathbf{g}+\mathbf{K}_{0},
$$

where $\mathbf{K}_{0}$ is a closed convex cone defined as $\mathbf{K}_{0}:=\left\{\mathbf{v} \in \mathbf{X} \mid \mathbf{v}_{\mathbf{n}} \leq 0\right.$ a.e. on $\left.\Gamma_{C}\right\}$.

Remark 2.5. Our definition of $\mathbf{K}$ differs from the usual one since we compute the gap in the direction of the normal $\mathbf{n}$ to the rigid foundation instead of the normal $\mathbf{n}_{\mathbf{o}}$ to $\partial \Omega$. Actually, under the small displacements hypothesis, the normal vector $\mathbf{n}$ and the gap $\mathbf{g}_{\mathbf{n}}$ to the rigid foundation can be replaced by $\mathbf{n}_{\mathbf{o}}$ and $\mathbf{g}_{\mathbf{n}_{\mathbf{o}}}$ (we refer 
to [20], Chap. 2 for the details). This ensures that in our context, using $\mathbf{n}_{\mathbf{o}}$ or $\mathbf{n}$ to write the formulation has no impact on the solution. However, it will be seen in the next sections that using $\mathbf{n}$ will be really convenient when dealing with shape optimization.

\subsection{Mathematical formulation of the problem}

Let us introduce the bilinear and linear forms $a: \mathbf{X} \times \mathbf{X} \rightarrow \mathbb{R}$ and $L: \mathbf{X} \rightarrow \mathbb{R}$, such that:

$$
a(\mathbf{u}, \mathbf{v}):=\int_{\Omega} \mathbb{C}: \boldsymbol{\epsilon}(\mathbf{u}): \boldsymbol{\epsilon}(\mathbf{v}), \quad L(\mathbf{v}):=\int_{\Omega} \mathbf{f} \mathbf{v}+\int_{\Gamma_{N}} \boldsymbol{\tau} \mathbf{v}
$$

According to the assumptions of the previous sections, one is able to show (see [4]) that $a$ is $\mathbf{X}$-elliptic with constant $\alpha_{0}$ (ellipticity of $\mathbb{C}$ and Korn's inequality), symmetric, continuous, and that $L$ is continuous (regularity of $\mathbf{f}$ and $\boldsymbol{\tau}$ ).

The unknown displacement $\mathbf{u}$ of the frictionless contact problem is the minimizer of the total mechanical energy of the elastic body, which reads, in the case of pure sliding (unilateral) contact problems:

$$
\inf _{\mathbf{v} \in \mathbf{K}} \varphi(\mathbf{v}):=\inf _{\mathbf{v} \in \mathbf{K}} \frac{1}{2} a(\mathbf{v}, \mathbf{v})-L(\mathbf{v}) .
$$

It is clear that the space $\mathbf{X}$, equipped with the usual $\mathbf{H}^{1}$ norm, is a Hilbert space. Moreover, under the conditions of the previous section, since $\mathbf{K}$ is obviously non-empty and the energy functional is strictly convex, continuous and coercive, we are able to conclude that $\mathbf{u}$ solution of (2.1) exists and is unique, see for example [9].

It is well known that (2.1) may be rewritten as a variational inequality (of the first kind):

$$
a(\mathbf{u}, \mathbf{v}-\mathbf{u}) \geq L(\mathbf{v}-\mathbf{u}), \quad \forall \mathbf{v} \in \mathbf{K} .
$$

Even if this variational inequality is very well known from the theoretical point of view such formulation is not well suited for computational purposes. One approach to get around this difficulty consists in rewriting the formulation so that the constraint is implicitly verified. One of the most frequently used formulation (in practical applications) is the penalty formulation. In [3], we suggested a method based on directional derivatives in order to derive optimality conditions for the penalty formulation.

The penalty formulation of (2.2) is numerically robust and relatively simple to implement, but its solutions depend on the penalty parameter. From a numerical point of view the simplest way to avoid this inconsistency while retaining the simplicity of the penalty approach is the augmented Lagrangian formulation. The rest of this work is related to the augmented Lagrangian formulation and the basic iterative process to solve this formulation, namely the augmented Lagrangian method.

It should be noticed that both formulations write as non-linear non-differentiable variational equations (possibly mixed), and thus lead to the same kind of technical difficulties (related to regularity) when studying the associated shape optimization problem. Here, an approach similar to [3] will be followed.

\subsection{Lagrangian formulation}

The content of this paragraph is based on [36]. The reader is referred to the chapter 4 of this monograph for all proofs and technical details. A mixed formulation of problem (2.1) can be recovered in the framework of Fenchel duality theory by deriving the dual problem associated to this minimization problem. This result is formally stated in the following theorem.

Theorem 2.6. If $\mathbf{u} \in \mathbf{K}$ is the solution of (2.1), then there exists a unique dual variable $\lambda \in H^{-\frac{1}{2}}\left(\Gamma_{C}\right)$ such that:

$$
a(\mathbf{u}, \mathbf{v})-L(\mathbf{v})+\left\langle\lambda, \mathbf{v}_{\mathbf{n}}\right\rangle_{\Gamma_{C}}=0, \quad \forall \mathbf{v} \in \mathbf{X}
$$




$$
\begin{aligned}
\langle\lambda, \zeta\rangle_{\Gamma_{C}} & \geq 0, \quad \forall \zeta \in H^{\frac{1}{2}}\left(\Gamma_{C}\right), \quad \zeta \geq 0, \\
\left\langle\lambda, \mathbf{u}_{\mathbf{n}}-\mathbf{g}_{\mathbf{n}}\right\rangle_{\Gamma_{C}} & =0 .
\end{aligned}
$$

Remark 2.7. It is important to note that, in general, the regularity of $\lambda$ is only $H^{-\frac{1}{2}}\left(\Gamma_{C}\right)$.

\subsection{Generalized Moreau-Yosida approximation}

The goal of this paragraph is to transform conditions (2.3b), (2.3c), into pointwise conditions using MoreauYosida regularizations. Since (2.1) is a non-smooth convex optimization problem, one may follow the approach from [18], Chapter 4 and introduce a consistent Moreau-Yosida regularization of (2.3), provided that the Lagrange multiplier belongs to a Hilbert space. From Theorem 2.6 this is not the case since $\lambda \in H^{-\frac{1}{2}}\left(\Gamma_{C}\right)$. However, it is known (see, e.g. [36], Chap. 4) that the additional regularity $\lambda \in L^{2}\left(\Gamma_{C}\right)$ can be obtained if the set $\left\{\mathbf{u}_{\mathbf{n}}-\mathbf{g}_{\mathbf{n}}=0\right\}$ is strictly contained in $\Gamma_{C}$, which can be formulated as follows:

Assumption 2.8. $\overline{\left\{\mathbf{u}_{\mathbf{n}}-\mathbf{g}_{\mathbf{n}}=0\right\}} \subset \Gamma_{C}$.

Remark 2.9. From the mechanical point of view, this assumption means that the points at the boundary of the potential contact zone $\Gamma_{C}$ do not come in contact with the rigid foundation. Intuitively, in the context of small displacements, it should be the case when $\Gamma_{C}$ is chosen large enough. Moreover, in practice, this assumption can be checked easily a posteriori.

From now on, it is assumed that Assumption 2.8 holds, thus, one may follow the steps of [18], Chapter 4 , which directly leads to the desired consistency result for the regularization. Before stating this result, we introduce a last notation, the projection onto $\mathbb{R}_{+}$in $\mathbb{R}$ (also called the positive part function) will be denoted $\mathrm{p}_{+}\left(\mathrm{p}_{+}(y):=\max \{0, y\}\right.$, for all $\left.y \in \mathbb{R}\right)$. We are now ready to introduce an Augmented Lagrangian Formulation (or characterization of the solution) of the sliding contact problem.

Theorem 2.10. Suppose Assumption 2.8 holds. If $(\mathbf{u}, \lambda) \in \mathbf{X} \times L^{2}\left(\Gamma_{C}\right)$ denotes the solution of (2.3), it verifies, for any $\gamma>0$,

$$
\begin{aligned}
a(\mathbf{u}, \mathbf{v})-L(\mathbf{v})+\left(\lambda, \mathbf{v}_{\mathbf{n}}\right)_{\Gamma_{C}} & =0, \quad \forall \mathbf{v} \in \mathbf{X} \\
\lambda-\mathrm{p}_{+}\left(\lambda+\gamma\left(\mathbf{u}_{\mathbf{n}}-\mathbf{g}_{\mathbf{n}}\right)\right) & =0 \quad \text { a.e. on } \Gamma_{C} .
\end{aligned}
$$

Conversely, if a pair $(\mathbf{u}, \lambda) \in \mathbf{X} \times L^{2}\left(\Gamma_{C}\right)$ satisfies (2.4) for some $\gamma>0$, then $\mathbf{u}$ is the solution of (2.1).

Proof. Let us start by rewriting (2.1) in a more suitable form. For this purpose, let $\Lambda \in \mathscr{L}\left(\mathbf{X}, L^{2}\left(\Gamma_{C}\right)\right)$ denote the normal trace operator, such that for all $\mathbf{v} \in \mathbf{X}$

$$
\Lambda \mathbf{v}=\mathbf{v}_{\mathbf{n}} .
$$

We also introduce the convex closed set $K:=\left\{\zeta \in L^{2}\left(\Gamma_{C}\right), \zeta \leq \mathbf{g}_{\mathbf{n}}\right.$ a.e. on $\left.\Gamma_{C}\right\}$. Using these notations, problem (2.1) rewrites as an unconstrained non-smooth convex optimization problem:

$$
\inf _{\mathbf{v} \in \mathbf{X}} \varphi(\mathbf{v})+I_{K}(\Lambda \mathbf{v})
$$

$I_{K}$ being non-smooth, we introduce $I_{K, \gamma}$ its generalized Moreau-Yosida regularization, with regularization parameter $\gamma>0$. Given $\zeta, \rho \in L^{2}\left(\Gamma_{C}\right)$, one has:

$$
I_{K, \gamma}(\zeta, \rho)=\inf _{\eta \in L^{2}\left(\Gamma_{C}\right)}\left\{I_{K}(\eta)+(\rho, \zeta-\eta)_{\Gamma_{C}}+\frac{\gamma}{2}\|\zeta-\eta\|_{0, \Gamma_{C}}^{2}\right\}
$$


Now, due to the indicator function in the inf, one gets:

$$
\begin{aligned}
I_{K, \gamma}(\zeta, \rho) & =\inf _{\eta \in K}\left\{\frac{\gamma}{2}\left\|\frac{\rho}{\gamma}+\zeta-\eta\right\|_{0, \Gamma_{C}}^{2}\right\}-\frac{1}{2 \gamma}\|\rho\|_{0, \Gamma_{C}}^{2} \\
& =\frac{\gamma}{2}\left\|\left(\mathrm{Id}-\operatorname{Proj}_{K}\right)\left(\frac{\rho}{\gamma}+\zeta\right)\right\|_{0, \Gamma_{C}}^{2}-\frac{1}{2 \gamma}\|\rho\|_{0, \Gamma_{C}}^{2} .
\end{aligned}
$$

Then, [18], Theorem 4.45 ensures that the complementarity conditions (2.3b), (2.3c) can equivalently be expressed as

$$
\lambda=\left.\frac{\partial}{\partial \zeta} I_{K, \gamma}(\zeta, \rho)\right|_{(\Lambda \mathbf{u}, \lambda)},
$$

for any $\gamma>0$, where the pair $(\mathbf{u}, \lambda)$ is the solution of (2.3). Moreover, due to definition of $K$, one has for any $\zeta \in L^{2}\left(\Gamma_{C}\right)$,

$$
\operatorname{Proj}_{K}(\zeta)=\min \left\{\zeta, \mathbf{g}_{\mathbf{n}}\right\}
$$

Therefore, condition (2.6) directly leads to the desired result.

\subsection{Augmented Lagrangian method}

The augmented Lagrangian method consists in an iterative algorithm to solve formulation (2.4) using an update strategy for the multiplier. The reader is referred to [10] for further details about the application of such methods to the numerical resolution of problems in mechanics. We briefly recall the steps of the algorithm.

\section{Algorithm: ALM}

1. Choose $\lambda^{0} \in L^{2}\left(\Gamma_{C}\right)$ and set $k=1$.

2. Choose $\gamma^{k}>0$, then find $\mathbf{u}^{k} \in \mathbf{X}$ the solution of,

$$
a\left(\mathbf{u}^{k}, \mathbf{v}\right)+\left(\mathrm{p}_{+}\left(\lambda^{k-1}+\gamma^{k}\left(\mathbf{u}_{\mathbf{n}}^{k}-\mathbf{g}_{\mathbf{n}}\right)\right), \mathbf{v}_{\mathbf{n}}\right)_{\Gamma_{C}}=L(\mathbf{v}) \quad \forall \mathbf{v} \in \mathbf{X},
$$

3. Update the multiplier following the rule:

$$
\lambda^{k}=\mathrm{p}_{+}\left(\lambda^{k-1}+\gamma^{k}\left(\mathbf{u}_{\mathbf{n}}^{k}-\mathbf{g}_{\mathbf{n}}\right)\right) \text { a.e. on } \Gamma_{C} .
$$

4. While a chosen convergence criterion is not satisfied, set $k=k+1$ and go back to step 2 .

It has been shown (see, e.g. in [36] for a detailed proof) that one gets the following convergence result for this algorithm.

Theorem 2.11. Suppose Assumption 2.8 holds, and $(\mathbf{u}, \lambda)$ denotes the solution of (2.4). Then for any choice of parameters $0<\gamma^{1} \leq \gamma^{2} \leq \cdots$, the iterates $\mathbf{u}^{k}$ converge to $\mathbf{u}$ strongly in $\mathbf{X}$. Moreover, the iterates of the multiplier $\lambda^{k}$ converge to $\lambda$ weakly in $L^{2}\left(\Gamma_{C}\right)$.

Remark 2.12. When $\lambda^{0} \in H^{\frac{1}{2}}\left(\Gamma_{C}\right)$, the iterates generated by the ALM satisfy $\left(\mathbf{u}^{k}, \lambda^{k}\right) \in \mathbf{X} \times H^{\frac{1}{2}}\left(\Gamma_{C}\right)$ for all $k \geq 1$. 


\section{SHAPE OPTIMIZATION}

Given a cost functional $J(\Omega)=\mathcal{J}(\Omega, \mathbf{u}(\Omega))$ depending explicitly on the domain $\Omega$, and also implicitly, through $\mathbf{u}(\Omega)$ the solution of (2.2) on $\Omega$, the optimization of $J$ with respect to $\Omega$ or shape optimization problem associated to the original contact formulation reads:

$$
\begin{cases}\text { minimize } & J(\Omega) \text { over } \Omega \in \mathcal{U}_{a d} \\ \text { subject to } & \mathbf{u}(\Omega) \text { solves }(2.2)\end{cases}
$$

where $\mathcal{U}_{a d}$ stands for the set of admissible domains.

Thanks to Theorem 2.11, from an analytical point of view, determining $\mathbf{u}$ and $\lambda$ using the ALM or any other method has limited impact on the computation of the shape sensitivity of (2.4) (or even (2.3)). The shape sensitivities would simply be defined relatively to the converged results of the ALM process. However, in practice, given a convergence criterion, the ALM will stop at some iteration $k$, meaning that even though $\mathbf{u}^{k}$ is assumed to be a good enough approximation of $\mathbf{u}$, it is not the solution of (2.4). Therefore, the sensitivities of (2.4) do not correspond to the sensitivities of the equations defining $\mathbf{u}^{k}$. As mentioned in the introduction, the idea in this work is to perform shape optimization on the approximate formulation (2.7) instead of the original formulation (2.1). Then, we look for a domain in $\mathcal{U}_{a d}$ that minimizes $J^{k}$, a cost functional defined by $J^{k}(\Omega):=\mathcal{J}\left(\Omega, \mathbf{u}^{k}(\Omega)\right)$ where $\mathbf{u}^{k}(\Omega)$ is the solution of (2.7) defined on $\Omega$. The new shape optimization is thus given by:

$$
\begin{cases}\text { minimize } & J^{k}(\Omega) \text { over } \Omega \in \mathcal{U}_{a d} \\ \text { subject to } & \mathbf{u}^{k}(\Omega) \text { solves }(2.7)\end{cases}
$$

Obviously, since we are interested in the optimality conditions associated to those problems, we also want to study the consistency of the sensitivities of the iterates $\mathbf{u}^{k}$ obtained from the ALM with respect to the sensitivity of $\mathbf{u}$.

Let $D \subset \mathbb{R}^{d}$ be a fixed bounded smooth domain, and let $\hat{\Gamma}_{D} \subset \partial D$ be a part of its boundary which will be the "potential" Dirichlet boundary. This means that for any domain $\Omega \subset D$, the Dirichlet boundary associated to $\Omega$ will be defined on $\Gamma_{D}:=\partial \Omega \cap \hat{\Gamma}_{D}$. With these notations, we introduce the set $\mathcal{U}_{a d}$ of all admissible domains, which consists of all smooth open domains $\Omega$ such that the Dirichlet boundary $\Gamma_{D} \subset \partial D$ is of strictly positive measure, that is:

$$
\mathcal{U}_{a d}:=\left\{\Omega \subset D \mid \Omega \text { is of class } \mathcal{C}^{1} \text { and }\left|\partial \Omega \cap \hat{\Gamma}_{D}\right|>0\right\}
$$

\subsection{Derivatives}

The shape optimization method followed in this work is the so-called perturbation of the identity, as presented in [26] and [16]. Let us introduce $\mathcal{C}_{b}^{1}\left(\mathbb{R}^{d}\right):=\left(\mathcal{C}^{1}\left(\mathbb{R}^{d}\right) \cap W^{1, \infty}\left(\mathbb{R}^{d}\right)\right)^{d}$, equipped with the $d$-dimensional $W^{1, \infty}$ norm, denoted $\|\cdot\|_{1, \infty}$. In order to move the domain $\Omega$, let $\boldsymbol{\theta} \in \mathcal{C}_{b}^{1}\left(\mathbb{R}^{d}\right)$ be a geometric deformation vector field. The associated perturbed or transported domain in the direction $\boldsymbol{\theta}$ will be defined as: $\Omega(t):=(\operatorname{Id}+t \boldsymbol{\theta})(\Omega)$ for any $t>0$. It is known that for $t$ sufficiently small, more specifically for $t$ such that $t\|\boldsymbol{\theta}\|_{1, \infty}<1$, Id $+t \boldsymbol{\theta}$ is a diffeomorphism, see for example [16]. This enables to rely on the classical notion of differentiability in Banach spaces to define shape differentiability. To make things clear some basic notions of shape sensitivity analysis from [34] are briefly recalled.

Let $y(\Omega)$ be the solution, in some Sobolev space denoted $W(\Omega)$, of a variational formulation posed on $\Omega$. For any fixed $\boldsymbol{\theta}$, for any small $t>0$, let $y(\Omega(t))$ be the solution of the same variational formulation posed on $\Omega(t)$. If the variational formulation is regular enough, which will be assumed to be true, it can be proved (see [34]) that $y(\Omega(t)) \circ(\operatorname{Id}+t \boldsymbol{\theta})$ also belongs to $W(\Omega)$. 
- The Lagrangian derivative or material derivative of $y(\Omega)$ in the direction $\boldsymbol{\theta}$ is the element $\dot{y}(\Omega)[\boldsymbol{\theta}] \in W(\Omega)$ defined by:

$$
\dot{y}(\Omega)[\boldsymbol{\theta}]:=\lim _{t \searrow 0} \frac{1}{t}(y(\Omega(t)) \circ(\operatorname{Id}+t \boldsymbol{\theta})-y(\Omega)) .
$$

If the limit is computed weakly in $W(\Omega)$ (respectively strongly), we talk about weak material derivative (respectively strong material derivative). Moreover, when the map $\boldsymbol{\theta} \mapsto \dot{y}(\Omega)[\boldsymbol{\theta}]$ is positively homogeneous, we will use the term conical material derivative.

- If the additional condition $\nabla y(\Omega) \boldsymbol{\theta} \in W(\Omega)$ holds for all $\boldsymbol{\theta} \in \mathcal{C}_{b}^{1}\left(\mathbb{R}^{d}\right)$, then one may define a directional derivative called the Eulerian derivative or shape derivative of $y(\Omega)$ in the direction $\boldsymbol{\theta}$ as the element $\mathrm{d} y(\Omega)[\boldsymbol{\theta}]$ of $W(\Omega)$ such that:

$$
\mathrm{d} y(\Omega)[\boldsymbol{\theta}]:=\dot{y}(\Omega)[\boldsymbol{\theta}]-\nabla y(\Omega) \boldsymbol{\theta}
$$

- The solution $y(\Omega)$ is said to be directionally shape differentiable if it admits a directional derivative for any admissible direction $\boldsymbol{\theta}$. If, in addition, the map $\boldsymbol{\theta} \mapsto \mathrm{d} y(\Omega)[\boldsymbol{\theta}]$ is positively homogeneous from $\boldsymbol{C}_{b}^{1}\left(\mathbb{R}^{d}\right)$ to $W(\Omega)$, $y(\Omega)$ is said to be conically shape differentiable. Furthermore, if this map is linear continuous from $\mathcal{C}_{b}^{1}\left(\mathbb{R}^{d}\right)$ to $W(\Omega), y(\Omega)$ is said to be shape differentiable.

Remark 3.1. Linearity and continuity of $\boldsymbol{\theta} \mapsto \dot{y}(\Omega)[\boldsymbol{\theta}]$ is actually equivalent to Gâteaux differentiability of the map $\boldsymbol{\theta} \mapsto y(\Omega(\boldsymbol{\theta})) \circ(\mathrm{Id}+\boldsymbol{\theta})$. The reader is referred to [6], Chapter 8 for a complete review on the different notions of derivatives.

Notation 3.2. Following the notations in [34], when there is no ambiguity, $y(\Omega)$ will be replaced simply by $y$, and for some fixed direction $\boldsymbol{\theta}$, we define $y_{t}:=y(\Omega(t)), y^{t}:=y_{t} \circ(\operatorname{Id}+t \boldsymbol{\theta})$. In the same way, the material and shape derivatives of $y$ at $\Omega$ in the direction $\boldsymbol{\theta}$ will be denoted $\dot{y}$ and $d y$, respectively.

\subsection{Shape sensitivity analysis of the original formulation}

In this section, we prove that the solution $\mathbf{u}$ to problem (2.1) admits conical material/shape derivatives in some specific directions $\boldsymbol{\theta}$. It is well known that $\mathbf{u}$ is not classically shape differentiable because the projection operator onto the closed convex $\mathbf{K}$ is not Fréchet-differentiable. However, it is known from [24] that projection operators are conically differentiable. Using this result, it has been proved in [33] that the Signorini problem admits conical material/shape derivatives in $2 \mathrm{~d}$ and $3 \mathrm{~d}$. Here, since formulation (2.1) is slightly different from the classical one due to our choice of $\mathbf{n}$ instead of $\mathbf{n}_{\mathbf{o}}$ for the contact, the proof in [33] needs to be adapted. Therefore, we will redo it, for some specific directions $\boldsymbol{\theta}$. Then, we derive sufficient conditions for the conical material/shape derivative of (2.1) to be solution of a more regular optimization problem.

Restriction on the directions $\boldsymbol{\theta}$. In view of the Zolésio-Hadamard structure theorem, we choose to limit ourselves to geometric deformation fields $\boldsymbol{\theta} \in \mathcal{C}_{b}^{1}\left(\mathbb{R}^{d}\right)$ along the direction of the normal $\mathbf{n}_{\mathbf{o}}$, i.e. vector fields of the form:

$$
\boldsymbol{\theta}=\theta \mathbf{n}_{\mathbf{o}}, \text { where } \theta \in \mathcal{C}_{b}^{1}\left(\mathbb{R}^{d}\right),
$$

where $\mathbf{n}_{\mathbf{o}}$ has been extended to $\mathcal{C}^{1}\left(\mathbb{R}^{d}\right)$, which is possible (see [16]) since $\partial \Omega$ is assumed to have $\mathcal{C}^{1}$ regularity. Moreover, this choice is well suited to our numerical algorithm, as it will be seen in the last section. The set of all these $\boldsymbol{\theta}$ is denoted $\boldsymbol{\Theta}$. Obviously, $\boldsymbol{\Theta}$ is a closed subspace of $\mathcal{C}_{b}^{1}\left(\mathbb{R}^{d}\right)$, thus it is a Banach space.

Let $\boldsymbol{\theta} \in \boldsymbol{\Theta}$ be a fixed direction. In order to perform sensitivity analysis with respect to the shape, let us first characterize $\mathbf{u}^{t}=\mathbf{u}_{t} \circ(\operatorname{Id}+t \boldsymbol{\theta})$ for $t>0$. This will be done by writing the problem solved by $\mathbf{u}_{t}$ on the transported domain $\Omega(t)$, then by bringing it back to $\Omega$ by a change of variables. Before that, some additional 
assumptions on the data are required. Indeed, since the domain is transported, the functions $\mathbb{C}, \mathbf{f}$ and $\boldsymbol{\tau}$ have to be defined everywhere in $\mathbb{R}^{d}$. They also need to enjoy more regularity for usual differentiability results to hold. In particular, we make the following regularity assumptions :

Assumption 3.3. $\mathbb{C} \in \mathcal{C}_{b}^{1}\left(\mathbb{R}^{d}, \mathbb{T}^{4}\right), \mathbf{f} \in \mathbf{H}^{1}\left(\mathbb{R}^{d}\right)$ and $\boldsymbol{\tau} \in \mathbf{H}^{2}\left(\mathbb{R}^{d}\right)$.

Notation 3.4. For the solution $\mathbf{u}_{t}$ to the transported problem on $\Omega(t)$, we introduce $\mathbf{X}_{t}:=\mathbf{H}_{\Gamma_{D}(t)}^{1}(\Omega(t))$ and the convex subset of admissible displacements:

$$
\mathbf{K}_{t}:=\left\{\mathbf{v} \in \mathbf{X}_{t} \mid \mathbf{v}_{\mathbf{n}} \leq \mathbf{g}_{\mathbf{n}} \text { a.e. on } \Gamma_{C}(t)\right\}
$$

Composition with the operator $\circ(\operatorname{Id}+t \boldsymbol{\theta})$ will be denoted by $(t)$, for instance, $\mathbb{C}(t):=\mathbb{C} \circ(\operatorname{Id}+t \boldsymbol{\theta})$. Besides, the normal component associated to $\mathbf{n}(t)$ of a vector $v$ is denoted $v_{\mathbf{n}(t)}$. For integral expressions, the Jacobian and tangential Jacobian of the transformation give $\mathrm{J}_{\Omega}(t):=\operatorname{Jac}(\operatorname{Id}+t \boldsymbol{\theta})$ and $\mathrm{J}_{\Gamma}(t):=\operatorname{Jac}_{\Gamma(t)}(\operatorname{Id}+t \boldsymbol{\theta})$. Finally, we introduce the transported strain tensor $\boldsymbol{\epsilon}^{t}$, the bilinear form $a^{t}$ on $\mathbf{X} \times \mathbf{X}$ and the linear form $L^{t}$ on $\mathbf{X}$, which are the versions of $\boldsymbol{\epsilon}, a$ and $L$ corresponding to the problem solved by $\mathbf{u}^{t}$, and are defined as follows:

$$
\begin{aligned}
a^{t}(\mathbf{z}, \mathbf{v}) & :=\int_{\Omega} \mathbb{C}(t): \boldsymbol{\epsilon}^{t}(\mathbf{z}): \boldsymbol{\epsilon}^{t}(\mathbf{v}) \mathrm{J}_{\Omega}(t) \quad \forall \mathbf{z}, \mathbf{v} \in \mathbf{X}, \\
\boldsymbol{\epsilon}^{t}(\mathbf{v}) & :=\frac{1}{2}\left(\nabla \mathbf{v}(\mathbf{I}+t \nabla \boldsymbol{\theta})^{-1}+\left(\mathbf{I}+t \nabla \boldsymbol{\theta}^{T}\right)^{-1} \nabla \mathbf{v}^{T}\right) \quad \forall \mathbf{v} \in \mathbf{X}, \\
L^{t}(\mathbf{v}) & :=\int_{\Omega} \mathbf{f}(t) \mathbf{v} \mathrm{J}_{\Gamma}(t)+\int_{\Gamma_{N}} \boldsymbol{\tau}(t) \mathbf{v} \mathrm{J}_{\Omega}(t) \quad \forall \mathbf{v} \in \mathbf{X} .
\end{aligned}
$$

Lemma 3.5. Let $\mathbf{v} \in \mathbf{X}_{t}$ and $\boldsymbol{\theta} \in \mathbf{\Theta}$, then $\mathbf{v} \in \mathbf{K}_{t}$ if and only if $\mathbf{v}(t) \in \mathbf{g}(t)+\mathbf{K}_{0}$.

Proof. The result is a direct consequence of our choice, (3.3), of direction $\boldsymbol{\Theta}$. Indeed, $\mathbf{n}=\mathbf{n}_{\mathbf{o}}$ on $\Gamma_{C}$ gives $\mathbf{n}(t)=\mathbf{n}$ on $\Gamma_{C}$. The rest follows from the definitions and the fact that $\operatorname{Id}+t \boldsymbol{\theta}$ is an isomorphism:

$$
\begin{aligned}
\mathbf{v} \in \mathbf{K}_{t} & \Longleftrightarrow(\mathbf{v}(y)-\mathbf{g}(y)) \cdot \mathbf{n}(y) \leq 0 \text { for a.e. } y \in \Gamma_{C}(t), \\
& \Longleftrightarrow(\mathbf{v}(x+t \boldsymbol{\theta}(x))-\mathbf{g}(x+t \boldsymbol{\theta}(x))) \cdot \mathbf{n}(x+t \boldsymbol{\theta}(x)) \leq 0 \text { for a.e. } x \in \Gamma_{C}, \\
& \Longleftrightarrow \mathbf{v}(t) \in \mathbf{g}(t)+\mathbf{K}_{0} .
\end{aligned}
$$

Remark 3.6. For $t$ sufficiently small, $\Gamma_{D}(t) \subset\left(\mathbb{R}^{d} \backslash \partial \Omega_{r i g}^{h^{\prime}}\right)$ and thus $\mathbf{g} \in \mathbf{X}_{t}$, which yields $\mathbf{g}(t) \in \mathbf{X}$.

From Lemma 3.5, it follows that $\mathbf{u}^{t}$ solves: find $\mathbf{u}^{t} \in \mathbf{g}(t)+\mathbf{K}_{0}$ such that,

$$
a^{t}\left(\mathbf{u}^{t}, \mathbf{v}-\mathbf{u}^{t}\right) \geq L^{t}\left(\mathbf{v}-\mathbf{u}^{t}\right), \quad \forall \mathbf{v} \in \mathbf{g}(t)+\mathbf{K}_{0} .
$$

Let us introduce the auxiliary unknowns $\mathbf{w}:=\mathbf{u}-\mathbf{g}$ and $\mathbf{w}^{t}:=\mathbf{u}^{t}-\mathbf{g}(t)$ which both belong to $\mathbf{K}_{0}$. Those functions satisfy the following variational inequalities:

$$
\begin{aligned}
a(\mathbf{w}, \mathbf{v}-\mathbf{w}) & \geq L(\mathbf{v}-\mathbf{w})-a(\mathbf{g}, \mathbf{v}-\mathbf{w}), \quad \forall \mathbf{v} \in \mathbf{K}_{0} \\
a^{t}\left(\mathbf{w}^{t}, \mathbf{v}-\mathbf{w}^{t}\right) & \geq L^{t}\left(\mathbf{v}-\mathbf{w}^{t}\right)-a^{t}\left(\mathbf{g}(t), \mathbf{v}-\mathbf{w}^{t}\right), \quad \forall \mathbf{v} \in \mathbf{K}_{0} .
\end{aligned}
$$

Before stating the conical differentiability result for $\mathbf{w}$ and $\mathbf{u}$, some additional notations are required. 
Notation 3.7. If $(\mathbf{u}, \lambda)$ denotes the solution of problem (2.4), let $\mathcal{A}, \mathcal{I}$ and $\mathcal{B}$ be the subsets of $\Gamma_{C}$ associated to the constraint $\mathbf{u} \in \mathbf{K}$ in problem (2.1):

$$
\begin{aligned}
& \mathcal{A}:=\left\{x \in \Gamma_{C}: \lambda(x)>0, \mathbf{u}_{\mathbf{n}}(x)-\mathbf{g}_{\mathbf{n}}(x)=0\right\}, \\
& \mathcal{I}:=\left\{x \in \Gamma_{C}: \lambda(x)=0, \mathbf{u}_{\mathbf{n}}(x)-\mathbf{g}_{\mathbf{n}}(x)<0\right\}, \\
& \mathcal{B}:=\left\{x \in \Gamma_{C}: \lambda(x)=0, \mathbf{u}_{\mathbf{n}}(x)-\mathbf{g}_{\mathbf{n}}(x)=0\right\}
\end{aligned}
$$

The subsets $\mathcal{A}, \mathcal{I}$ and $\mathcal{B}$ are usually referred to as the active, inactive and biactive sets. Note that they are at least measurable due to the regularities of $\lambda, \mathbf{u}$ and $\mathbf{g}_{\mathbf{n}}$. Moreover, we only consider cases where contact occurs, which means that $\mathcal{A}$ is non-empty.

The bilinear form $a^{\prime}$ and the linear forms $\boldsymbol{\epsilon}^{\prime}, L^{\prime}$, which will be naturally appear when differentiating (3.4b) with respect to the shape, are introduced: for any $\mathbf{u}, \mathbf{v} \in \mathbf{X}$,

$$
\begin{aligned}
& a^{\prime}(\mathbf{u}, \mathbf{v}):=\int_{\Omega}\left\{\mathbb{C}: \boldsymbol{\epsilon}^{\prime}(\mathbf{u}): \boldsymbol{\epsilon}(\mathbf{v})+\mathbb{C}: \epsilon(\mathbf{u}): \boldsymbol{\epsilon}^{\prime}(\mathbf{v})+(\operatorname{div} \boldsymbol{\theta} \mathbb{C}+\nabla \mathbb{C} \boldsymbol{\theta}): \boldsymbol{\epsilon}(\mathbf{u}): \boldsymbol{\epsilon}(\mathbf{v})\right\}, \\
& \boldsymbol{\epsilon}^{\prime}(\mathbf{v}):=-\frac{1}{2}\left(\nabla \mathbf{v} \nabla \boldsymbol{\theta}+\nabla \boldsymbol{\theta}^{T} \nabla \mathbf{v}^{T}\right) \\
& L^{\prime}(\mathbf{v})=\int_{\Omega}(\operatorname{div} \boldsymbol{\theta} \mathbf{f}+\nabla \mathbf{f} \boldsymbol{\theta}) \mathbf{v}+\int_{\Gamma_{N}}\left(\operatorname{div}_{\Gamma} \boldsymbol{\theta} \boldsymbol{\tau}+\nabla \boldsymbol{\tau} \boldsymbol{\theta}\right) \mathbf{v}
\end{aligned}
$$

Moreover, for any smooth function $f$ defined on $\mathbb{R}^{d}$, and that does not depend on $\Omega$, we denote $f^{\prime}[\boldsymbol{\theta}]$ or simply $f^{\prime}$ the following directional derivative:

$$
f^{\prime}[\boldsymbol{\theta}]:=\lim _{t \searrow 0} \frac{1}{t}(f(t)-f)=(\nabla f) \boldsymbol{\theta} .
$$

Using this notation, $\mathbf{n}^{\prime}:=(\nabla \mathbf{n}) \boldsymbol{\theta}$ and for any $\mathbf{v} \in \mathbf{X}$ one has $\mathbf{v}_{\mathbf{n}^{\prime}}:=\mathbf{v} \cdot \mathbf{n}^{\prime}$. For the gap $\left(\mathbf{g}_{\mathbf{n}}\right)^{\prime}:=\left(\nabla \mathbf{g}_{\mathbf{n}}\right) \boldsymbol{\theta}$ and since $\mathbf{g}_{\mathbf{n}}$ is the oriented distance function to the smooth boundary $\partial \Omega_{\text {rig }}, \nabla \mathbf{g}_{\mathbf{n}}=-\mathbf{n}$, which implies that $\left(\mathbf{g}_{\mathbf{n}}\right)^{\prime}=-\boldsymbol{\theta} \cdot \mathbf{n}$. However, we will still use the notation $\left(\mathbf{g}_{\mathbf{n}}\right)^{\prime}$ to emphasize that this term comes from differentiation of the gap.

Remark 3.8. From the definition of $\boldsymbol{\Theta}$, one gets that any $\boldsymbol{\theta} \in \boldsymbol{\Theta}$ satisfies $\boldsymbol{\theta}=\theta \mathbf{n}$ on $\Gamma_{C}$. This implies that:

$$
\mathbf{n}^{\prime}:=(\nabla \mathbf{n}) \boldsymbol{\theta}=\theta(\nabla \mathbf{n}) \mathbf{n}=0 \quad \text { on } \Gamma_{C}
$$

since $\mathbf{n}$ is a unit vector. Therefore, one automatically gets for the gap:

$$
\left(\mathbf{g}_{\mathbf{n}}\right)^{\prime}=(\mathbf{g} \cdot \mathbf{n})^{\prime}=\mathbf{g}^{\prime} \cdot \mathbf{n}+\mathbf{g} \cdot \mathbf{n}^{\prime}=\mathbf{g}^{\prime} \cdot \mathbf{n} \quad \text { on } \Gamma_{C} .
$$

Thus, using the notations introduced earlier, it is possible to replace $\left(\mathbf{g}_{\mathbf{n}}\right)^{\prime}$ by $\mathbf{g}_{\mathbf{n}}^{\prime}=\mathbf{g}^{\prime} \cdot \mathbf{n}$ on $\Gamma_{C}$.

We can now use some of the results on the conical differentiability of projections on closed convex sets. In the next two results, material derivatives will be characterized using some concepts of capacity theory. More precisely the cones containing those derivatives will be defined up to a set of zero capacity (denoted q.e. for quasi-everywhere). The reader is referred to [24] and [7] for further details about capacity theory.

Theorem 3.9. Under Assumption 3.3, the solution $\mathbf{w}$ of (3.4a) is conically shape differentiable on $\boldsymbol{\Theta}$ and its conical material derivative in the direction $\boldsymbol{\theta} \in \boldsymbol{\Theta}$ is given by the solution of the problem: find $\dot{\mathbf{w}} \in S^{\mathbf{w}}\left(\mathbf{K}_{0}\right)$ such that,

$$
a(\dot{\mathbf{w}}, \boldsymbol{\phi}-\dot{\mathbf{w}}) \geq L^{\prime}(\boldsymbol{\phi}-\dot{\mathbf{w}})-a^{\prime}(\mathbf{u}, \boldsymbol{\phi}-\dot{\mathbf{w}})-a\left(\mathbf{g}^{\prime}, \boldsymbol{\phi}-\dot{\mathbf{w}}\right), \quad \forall \boldsymbol{\phi} \in S^{\mathbf{w}}\left(\mathbf{K}_{0}\right),
$$


Moreover, one has the characterization $S^{\mathbf{w}}\left(\mathbf{K}_{0}\right)=\left\{\phi \in \mathbf{X} \mid \phi_{\mathbf{n}} \leq 0\right.$ q.e. on $\mathcal{A} \cup \mathcal{B}$ and $\left.a(\mathbf{u}, \phi)=L(\phi)\right\}$.

Proof. We follow the same steps as in [23], Section 5.2, and adapt each of them to our specific formulation. The idea is to write $\mathbf{w}^{t}$ as the projection onto $\mathbf{K}_{0}$ of some element in $\mathbf{X}$. Then, using the conical differentiability of the projection (see [24]), one is able to perform a first order expansion of $\mathbf{w}^{t}$ around $t=0^{+}$.

First, let us prove strong continuity of the map $t \mapsto \mathbf{w}^{t}$ at $t=0^{+}$in $\mathbf{X}$. Taking respectively $\mathbf{v}=\mathbf{w}$ and $\mathbf{v}=\mathbf{w}^{t}$ as test-functions in (3.4b) and (3.4a), then adding the two formulations, one obtains

$$
a\left(\mathbf{w}-\mathbf{w}^{t}, \mathbf{w}-\mathbf{w}^{t}\right) \leq\left(L^{t}-L\right)\left(\mathbf{w}-\mathbf{w}^{t}\right)-a^{t}\left(\mathbf{g}(t), \mathbf{w}-\mathbf{w}^{t}\right)+a\left(\mathbf{g}, \mathbf{w}-\mathbf{w}^{t}\right) .
$$

Using ellipticity of $a$, one deduces the following estimation:

$$
\alpha_{0}\left\|\mathbf{w}-\mathbf{w}^{t}\right\|_{\mathbf{X}} \leq\left\|L^{t}-L\right\|_{\mathbf{X}^{*}}+C\left(\left\|a^{t}-a\right\|+\|\mathbf{g}(t)-\mathbf{g}\|_{\mathbf{X}}\right)
$$

which yields the result since the right hand side goes to 0 , due to the properties of $a^{t}, L^{t}$, and the regularity of g. Next, using this result and again the properties of $a^{t}, L^{t}$, we proceed to the expansion of each term in (3.4b), which leads to:

$$
\begin{aligned}
a^{t}\left(\mathbf{w}^{t}, \mathbf{v}-\mathbf{w}^{t}\right) & =a\left(\mathbf{w}^{t}, \mathbf{v}-\mathbf{w}^{t}\right)+t a^{\prime}\left(\mathbf{w}, \mathbf{v}-\mathbf{w}^{t}\right)+o(t) \\
L^{t}\left(\mathbf{v}-\mathbf{w}^{t}\right) & =L\left(\mathbf{v}-\mathbf{w}^{t}\right)+t L^{\prime}\left(\mathbf{v}-\mathbf{w}^{t}\right)+o(t) \\
a^{t}\left(\mathbf{g}(t), \mathbf{v}-\mathbf{w}^{t}\right) & =a\left(\mathbf{g}, \mathbf{v}-\mathbf{w}^{t}\right)+t a^{\prime}\left(\mathbf{g}, \mathbf{v}-\mathbf{w}^{t}\right)+t a\left(\mathbf{g}^{\prime}, \mathbf{v}-\mathbf{w}^{t}\right)+o(t) .
\end{aligned}
$$

Plugging these expansions in (3.4b) yields: for all $\mathbf{v} \in \mathbf{K}_{0}$,

$$
a\left(\mathbf{w}^{t}, \mathbf{v}-\mathbf{w}^{t}\right) \geq L\left(\mathbf{v}-\mathbf{w}^{t}\right)-a\left(\mathbf{g}, \mathbf{v}-\mathbf{w}^{t}\right)+t\left(L^{\prime}\left(\mathbf{v}-\mathbf{w}^{t}\right)-a^{\prime}\left(\mathbf{u}, \mathbf{v}-\mathbf{w}^{t}\right)-a\left(\mathbf{g}^{\prime}, \mathbf{v}-\mathbf{w}^{t}\right)\right)+o(t) .
$$

Let $\mathfrak{l}, \mathfrak{l}^{\prime}, \mathfrak{a}_{\mathbf{u}}^{\prime} \in \mathbf{X}$ such that for all $\mathbf{z} \in \mathbf{X}$ :

$$
a(\mathfrak{l}, \mathbf{z})=L(\mathbf{z}), \quad a\left(\mathfrak{l}^{\prime}, \mathbf{z}\right)=L^{\prime}(\mathbf{z}), \quad a\left(\mathfrak{a}_{\mathbf{u}}^{\prime}, \mathbf{z}\right)=a^{\prime}(\mathbf{u}, \mathbf{z}) .
$$

Then relation (3.7) can be equivalently rewritten as:

$$
\begin{aligned}
\mathbf{w}^{t} & =\operatorname{Proj}_{\mathbf{K}_{0}}^{a}\left(\mathfrak{l}-\mathbf{g}+t\left(\mathfrak{l}^{\prime}-\mathfrak{a}_{\mathbf{u}}^{\prime}-\mathbf{g}^{\prime}\right)+o(t)\right) \\
& =\operatorname{Proj}_{\mathbf{K}_{0}}^{a}\left(\mathfrak{l}-\mathbf{g}+t\left(\mathfrak{l}^{\prime}-\mathfrak{a}_{\mathbf{u}}^{\prime}-\mathbf{g}^{\prime}\right)\right)+o(t) .
\end{aligned}
$$

As seen in Remark 2.5, one has the approximation $\mathbf{n}=\mathbf{n}_{\mathbf{o}}$ on $\Gamma_{C}$ under the small displacements hypothesis. Thus we directly get from [23], Lemma 5.2.9 that $\mathbf{K}_{0}$ is polyhedric. Therefore, from Theorem 2.4, it follows that $\operatorname{Proj}_{\mathbf{K}_{0}}^{a}$ is conically differentiable and that:

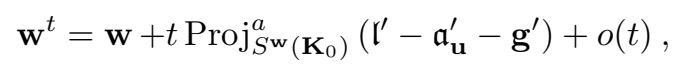

which yields (3.6). Finally, the characterization of $S^{\mathbf{w}}\left(\mathbf{K}_{0}\right)$ is also given by [23], Lemma 5.2.9.

Corollary 3.10. Under Assumption 3.3, the solution $\mathbf{u}$ of (2.1) is conically shape differentiable on $\boldsymbol{\Theta}$ and its conical material derivative in any direction $\boldsymbol{\theta} \in \mathbf{\Theta}$ is given by the solution of: find $\dot{\mathbf{u}} \in \mathbf{S}$ such that,

$$
a(\dot{\mathbf{u}}, \boldsymbol{\psi}-\dot{\mathbf{u}}) \geq L^{\prime}(\boldsymbol{\psi}-\dot{\mathbf{u}})-a^{\prime}(\mathbf{u}, \boldsymbol{\psi}-\dot{\mathbf{u}}), \quad \forall \boldsymbol{\psi} \in \mathbf{S},
$$

where $\mathbf{S}:=\mathbf{g}^{\prime}+S^{\mathbf{w}}\left(\mathbf{K}_{0}\right)=\left\{\boldsymbol{\psi} \in \mathbf{g}^{\prime}+\mathbf{X} \mid \boldsymbol{\psi}_{\mathbf{n}} \leq \mathbf{g}_{\mathbf{n}}^{\prime}\right.$ q.e. on $\mathcal{A} \cup \mathcal{B}$ and $\left.a\left(\mathbf{u}, \boldsymbol{\psi}-\mathbf{g}^{\prime}\right)=L\left(\boldsymbol{\psi}-\mathbf{g}^{\prime}\right)\right\}$.

Proof. This is a direct consequence of Theorem 3.9 since $\mathbf{u}^{t}=\mathbf{w}^{t}+\mathbf{g}(t)$ and therefore $\dot{\mathbf{u}}=\dot{\mathbf{w}}+\mathbf{g}^{\prime}$. 
As formulations (3.6) and (3.8) relies on zero capacity sets they are not easy to handle. However, under some mild additional regularity assumptions, it is possible to rewrite those formulations as standard optimization problems. In the same way as in [17], Section 4.1 for the obstacle problem, we introduce the following regularity assumption:

Assumption 3.11. $\mathcal{A} \cup \mathcal{B}=\overline{\operatorname{int}(\mathcal{A} \cup \mathcal{B})}$.

Remark 3.12. This assumption implies that not only $\mathcal{A} \cup \mathcal{B}$ is closed, but also it has a non-empty interior. Moreover, for every $\phi \in \mathbf{X}$ it is obvious, that

$$
\phi_{\mathbf{n}} \leq 0 \text { q.e. on } \operatorname{int}(\mathcal{A} \cup \mathcal{B}) \Longrightarrow \phi_{\mathbf{n}} \leq 0 \text { a.e. on } \operatorname{int}(\mathcal{A} \cup \mathcal{B})
$$

Regarding the converse implication, we invoke the result stated in [34], Lemma 4.31, from which we get that the subspace

$$
\mathcal{H}:=\left\{\phi_{\mathbf{n}} \in H^{\frac{1}{2}}\left(\Gamma_{C}\right) \mid \phi \in \mathbf{X}\right\}
$$

associated with the appropriate bilinear form (expressed in [34], Eq. (4.192)) is a Dirichlet space in the sense of [24], Definition 3.1. Therefore, $\phi_{\mathbf{n}}$ admits a unique quasi-continuous representative in the equivalence class related to the "q.e. on $\Gamma_{C}$ equality". Considering this specific representative, one gets from [7], Theorem 5 that

$$
\phi_{\mathbf{n}} \leq 0 \text { a.e. on } \operatorname{int}(\mathcal{A} \cup \mathcal{B}) \Longrightarrow \phi_{\mathbf{n}} \leq 0 \text { q.e. on } \operatorname{int}(\mathcal{A} \cup \mathcal{B}) \text {, }
$$

since $\operatorname{int}(\mathcal{A} \cup \mathcal{B})$ is an open subset of $\Gamma_{C}$. Quasi-continuity of $\phi_{\mathbf{n}}$ on $\Gamma_{C}$ also implies that $\phi_{\mathbf{n}} \leq 0$ q.e. on $\overline{\operatorname{int}(\mathcal{A} \cup \mathcal{B})}=\mathcal{A} \cup \mathcal{B}$.

Before stating the optimization problem that $\dot{\mathbf{w}}$ solves when Assumption 3.11 is fulfilled, let us define the subspace $\mathbf{X}_{\mathcal{A}} \subset \mathbf{X}$, and the cone $\mathbf{K}_{\mathcal{A}}$ such that:

$$
\mathbf{X}_{\mathcal{A}}:=\left\{\phi \in \mathbf{X} \mid \phi_{\mathbf{n}}=0 \text { a.e. on } \mathcal{A}\right\}, \quad \mathbf{K}_{\mathcal{A}}:=\left\{\phi \in \mathbf{X}_{\mathcal{A}} \mid \phi_{\mathbf{n}} \leq 0 \text { a.e. on } \mathcal{B}\right\} .
$$

Clearly, $\mathbf{X}_{\mathcal{A}}$ is a closed subspace of $\mathbf{X}$, therefore it is a Hilbert space, and $\mathbf{K}_{\mathcal{A}}$ is a non-empty closed convex cone.

Theorem 3.13. If Assumptions 3.3 and 3.11 hold, then $\dot{\mathbf{w}}$ is the solution of (3.6) if and only if it solves:

$$
\inf _{\phi \in \mathbf{K}_{\mathcal{A}}} \frac{1}{2} a(\phi, \phi)-L^{\prime}(\phi)+a^{\prime}(\mathbf{u}, \phi)+a\left(\mathbf{g}^{\prime}, \boldsymbol{\phi}\right) .
$$

Proof. It suffices to prove that $S^{\mathbf{w}}\left(\mathbf{K}_{0}\right)=\mathbf{K}_{\mathcal{A}}$. First of all, let us point out that in the characterization of $S^{\mathbf{w}}\left(\mathbf{K}_{0}\right)$ given in Theorem 3.9, since $\phi \in \mathbf{X}$ and $\mathbf{u}$ solves $(2.4 \mathrm{a})$, condition $a(\mathbf{u}, \phi)=L(\phi)$ is equivalent to $\left(\lambda, \phi_{\mathbf{n}}\right)_{\Gamma_{C}}=0$. As $\lambda \in L^{2}\left(\Gamma_{C}\right), \lambda>0$ on $\mathcal{A}$ and $\lambda=0$ on $\Gamma_{C} \backslash \mathcal{A}$ it follows that:

$$
\left(\phi \in \mathbf{X} \text { s.t. } \phi_{\mathbf{n}} \leq 0 \text { a.e. on } \mathcal{A} \cup \mathcal{B} \text { and }\left(\lambda, \phi_{\mathbf{n}}\right)_{\Gamma_{C}}=0\right) \Longleftrightarrow \phi \in \mathbf{K}_{\mathcal{A}} \text {. }
$$

And Remark 3.12 gives us the equality of both sets.

Finally, some obvious results are obtained, introducing the following assumption.

Assumption 3.14. The biactive set $\mathcal{B}$ is of measure zero with respect to $\Gamma_{C}(\mathrm{a}(d-1)$-dimensional submanifold of $\mathbb{R}^{d}$ ). 
Remark 3.15. Assumptions on the biactive set are often made when considering optimal control problems for variational inequalities (see, e.g. [17]) because this is the set of points where non-differentiabilities occur. From the mechanical point of view, a point $x \in \mathcal{B}$ is a point such that $\mathbf{u}_{\mathbf{n}}(x)=\mathbf{g}_{\mathbf{n}}(x)$ and $\lambda(x)=0$, that is $x$ is in contact but there is no contact pressure. The set $\mathcal{B}$ is often referred to as the set of weak contact points. For example, Assumption 3.14 is verified when all weak contact points represent a countable number of point in 2D or a countable number of curves in $3 \mathrm{D}$.

Corollary 3.16. If, in addition to the assumptions of Theorem 3.13, Assumption 3.14 holds, then $\dot{\mathbf{w}}$ is the solution of (3.6) if and only if it solves: find $\dot{\mathbf{w}} \in \mathbf{X}_{\mathcal{A}}$ such that

$$
a(\dot{\mathbf{w}}, \phi)=L^{\prime}(\phi)-a^{\prime}(\mathbf{u}, \phi)-a\left(\mathbf{g}^{\prime}, \phi\right), \quad \forall \phi \in \mathbf{X}_{\mathcal{A}}
$$

Proof. When $\mathcal{B}$ is of measure zero, $\mathbf{K}_{\mathcal{A}}=\mathbf{X}_{\mathcal{A}}$ and the result follows as problem (3.9) can be equivalently rewritten as a linear variational formulation.

Remark 3.17. Under the assumption of Corollary 3.16, the material derivative becomes linear with respect to $\boldsymbol{\theta}$, which implies that $\mathbf{u}$ is strongly shape differentiable in $L^{2}(\Omega)$, and its shape derivative in any direction $\boldsymbol{\theta} \in \boldsymbol{\Theta}$ is given by:

$$
d \mathbf{u}=\dot{\mathbf{u}}-\nabla \mathbf{u} \boldsymbol{\theta}=\dot{\mathbf{w}}+\mathbf{g}^{\prime}-\nabla \mathbf{u} \boldsymbol{\theta}
$$

where $\dot{\mathbf{w}} \in \mathbf{X}_{\mathcal{A}}$ is the unique solution of (3.10). Note that this conclusion is similar to the one in [27], Remark 4.1.

\subsection{Shape sensitivity analysis of the augmented Lagrangian formulation}

The goal of this section is to prove, at every iteration of the ALM algorithm, the differentiability of $\mathbf{u}^{k}$ with respect to the shape. As $\mathrm{p}_{+}$fails to be Frêchet differentiable it is not possible to rely on the implicit function theorem as in [16]. However, as it is a projection operator, it is conically differentiable, which enables us to show existence of conical material/shape derivatives for $\mathbf{u}^{k}$ following the approach in [34]. Then, under assumptions on some specific subsets of $\Gamma_{C}$ (this will be presented and referred to as Assumption 3.23), classical shape differentiability of $\mathbf{u}^{k}$ is proved.

First of all, let us briefly recall some properties of function $\mathrm{p}_{+}$.

Lemma 3.18. The function $\mathrm{p}_{+}: \mathbb{R} \rightarrow \mathbb{R}$ is Lipschitz continuous and conically differentiable, with conical derivative at $u$ in the direction $v \in \mathbb{R}$ :

$$
d \mathrm{p}_{+}(u ; v)= \begin{cases}0 & \text { if } u<0 \\ \mathrm{p}_{+}(v) & \text { if } u=0 \\ v & \text { if } u>0\end{cases}
$$

Lemma 3.19. The Nemytskij operator $\mathrm{p}_{+}: L^{2}\left(\Gamma_{C}\right) \rightarrow L^{2}\left(\Gamma_{C}\right)$ is Lipschitz continuous and conically differentiable.

Remark 3.20. These results are well known if "conically" is replaced by "directionally", a proof can be found in [37], for example. Conical differentiability then follows directly because for any $u \in \mathbb{R}, d \mathrm{p}_{+}(u ; \cdot): \mathbb{R} \rightarrow \mathbb{R}$ is obviously positively homogeneous, and the same property holds for $d \mathrm{p}_{+}(u ; \cdot): L^{2}\left(\Gamma_{C}\right) \rightarrow L^{2}\left(\Gamma_{C}\right)$ for any $u \in L^{2}\left(\Gamma_{C}\right)$.

Theorem 3.21. If Assumption 3.3 holds and $\lambda^{0} \in H^{\frac{3}{2}}\left(\Gamma_{C}\right)$, then for any $k \geq 0$, the pair $\left(\mathbf{u}^{k}, \lambda^{k}\right)$ defined by (2.7)-(2.8) is conically shape differentiable on $\boldsymbol{\Theta}$, strongly in $\mathbf{X} \times L^{2}\left(\Gamma_{C}\right)$. 
Proof. As the solution of (2.7) at each step $k+1$ depends on the previous iterate, the result will be proved by induction.

Base case For $k=0$, given $\gamma^{1}>0$, the first iteration of the ALM gives $\mathbf{u}^{1} \in \mathbf{X}$ the solution of

$$
a\left(\mathbf{u}^{1}, \mathbf{v}\right)+\left(R_{\mathbf{n}}^{1}\left(\mathbf{u}^{1}\right), \mathbf{v}_{\mathbf{n}}\right)_{\Gamma_{C}}=L(\mathbf{v}),
$$

where we have introduced the non-linear map $R_{\mathbf{n}}^{1}: \mathbf{X} \rightarrow H^{\frac{1}{2}}\left(\Gamma_{C}\right)$ defined as,

$$
R_{\mathbf{n}}^{1}(\mathbf{v}):=\mathrm{p}_{+}\left(\lambda^{0}+\gamma^{1}\left(\mathbf{v}_{\mathbf{n}}-\mathbf{g}_{\mathbf{n}}\right)\right) \quad \forall \mathbf{v} \in \mathbf{X}
$$

Obviously, $R_{\mathbf{n}}^{1}\left(\mathbf{u}^{1}\right)=\lambda^{1}$, but we prefer here to denote $R_{\mathbf{n}}^{1}\left(\mathbf{u}^{1}\right)$ to emphasize that $\lambda^{1}$ is defined explicitly from $\mathbf{u}^{1}$ and the data. Using the same arguments as in [3], one gets that, due to the regularity of the data, especially $\lambda^{0}, \mathbf{u}^{1}$ admits a strong material derivative $\dot{\mathbf{u}}^{1} \in \mathbf{X}$ in each direction $\boldsymbol{\theta} \in \boldsymbol{\Theta}$, which is the unique solution of

$$
a\left(\dot{\mathbf{u}}^{1}, \mathbf{v}\right)+\left(R_{\mathbf{n}}^{1^{\prime}}\left(\mathbf{u}^{1}\right), \mathbf{v}_{\mathbf{n}}\right)_{\Gamma_{C}}=L^{1}[\boldsymbol{\theta}](\mathbf{v})
$$

where, for any $\mathbf{v} \in \mathbf{X}$, with material derivative $\dot{\mathbf{v}} \in \mathbf{X}$, we have

$$
\begin{aligned}
R_{\mathbf{n}}^{1^{\prime}}(\mathbf{v}) & :=d \mathbf{p}_{+}\left(\lambda^{0}+\gamma^{1}\left(\mathbf{v}_{\mathbf{n}}-\mathbf{g}_{\mathbf{n}}\right) ;\left(\lambda^{0}\right)^{\prime}+\gamma^{1}\left(\dot{\mathbf{v}}_{\mathbf{n}}-\mathbf{g}_{\mathbf{n}}^{\prime}\right)\right) \\
L^{1}[\boldsymbol{\theta}](\mathbf{v}) & :=L^{\prime}(\mathbf{v})-a^{\prime}\left(\mathbf{u}^{1}, \mathbf{v}\right)-\int_{\Gamma_{C}} \lambda^{1} \mathbf{v}_{\mathbf{n}} \operatorname{div}_{\Gamma} \boldsymbol{\theta}
\end{aligned}
$$

where the notation introduced in (3.5) has been used for $\left(\lambda^{0}\right)^{\prime}$. Moreover, as $\lambda^{1}$ only depends on $\lambda^{0}$ and $\mathbf{u}^{1}$, it is clear that it also admits a strong material derivative in $L^{2}\left(\Gamma_{C}\right)$ in the direction $\boldsymbol{\theta}$, which is simply obtained by differentiating (2.8):

$$
\dot{\lambda}^{1}=R_{\mathbf{n}}^{1^{\prime}}\left(\mathbf{u}^{1}\right),
$$

which finishes to prove that $\left(\mathbf{u}^{1}, \lambda^{1}\right)$ is directionally shape differentiable. Moreover, one has that $a$ is bilinear, the maps $\boldsymbol{\theta} \mapsto L^{1}[\boldsymbol{\theta}],\left(\lambda^{0}\right)^{\prime}, \mathbf{g}_{\mathbf{n}}^{\prime}$ are linear and $d \mathrm{p}_{+}\left(\lambda^{0}+\gamma^{1}\left(\mathbf{u}_{\mathbf{n}}^{1}-\mathbf{g}_{\mathbf{n}}\right) ; \cdot\right)$ is positively homogeneous. Thus one deduces that the map $\boldsymbol{\theta} \mapsto \dot{\mathbf{u}}^{1}$ is positively homogeneous. The same property holds for $\boldsymbol{\theta} \mapsto \dot{\lambda}^{1}$ due to (3.11).

Inductive step Let $k \geq 1$ and assume $\left(\mathbf{u}^{k}, \lambda^{k}\right)$ is conically shape differentiable, strongly in $\mathbf{X} \times L^{2}\left(\Gamma_{C}\right)$. This implies that $\lambda^{k}$ admits a strong material derivative $\dot{\lambda}^{k} \in L^{2}\left(\Gamma_{C}\right)$. Hence, using the arguments of the case $k=0$, one may differentiate (2.7), which leads to existence of a unique material derivative $\dot{\mathbf{u}}^{k+1} \in \mathbf{X}$ for $\mathbf{u}^{k+1}$ that solves:

$$
a\left(\dot{\mathbf{u}}^{k+1}, \mathbf{v}\right)+\left(R_{\mathbf{n}}^{k+1^{\prime}}\left(\mathbf{u}^{k+1}\right), \mathbf{v}_{\mathbf{n}}\right)_{\Gamma_{C}}=L^{k+1}[\boldsymbol{\theta}](\mathbf{v}),
$$

where the same notation as in the case $k=0$ has been used for $L^{k+1}[\boldsymbol{\theta}]$, while the notation for $R_{\mathbf{n}}^{k+1^{\prime}}$ has been slightly adapted:

$$
R_{\mathbf{n}}^{k+1^{\prime}}(\mathbf{v}):=d \mathbf{p}_{+}\left(\lambda^{k}+\gamma^{k+1}\left(\mathbf{v}_{\mathbf{n}}-\mathbf{g}_{\mathbf{n}}\right) ; \dot{\lambda}^{k}+\gamma^{k+1}\left(\dot{\mathbf{v}}_{\mathbf{n}}-\mathbf{g}_{\mathbf{n}}^{\prime}\right)\right)
$$


Especially, this also proves that $\lambda^{k+1}$ admits a material derivative in $L^{2}\left(\Gamma_{C}\right)$ defined as

$$
\dot{\lambda}^{k+1}=R_{\mathbf{n}}^{k+1^{\prime}}\left(\mathbf{u}^{k+1}\right) .
$$

Again, since $a$ is bilinear, $\boldsymbol{\theta} \mapsto L^{k+1}[\boldsymbol{\theta}], \mathbf{g}_{\mathbf{n}}^{\prime}$ are linear, and $d \mathrm{p}_{+}\left(\lambda^{k}+\gamma^{k+1}\left(\mathbf{u}_{\mathbf{n}}^{k+1}-\mathbf{g}_{\mathbf{n}}\right) ; \cdot\right), \boldsymbol{\theta} \mapsto \dot{\lambda}^{k}$ are positively homogeneous, one deduces that $\boldsymbol{\theta} \mapsto\left(\dot{\mathbf{u}}^{k+1}, \dot{\lambda}^{k+1}\right)$ is positively homogeneous as well.

Remark 3.22. Existence of directional (even conical) material/shape derivatives in all directions does not guarantee that the solution $\mathbf{u}^{k}$ is shape differentiable. Indeed, as these derivative depends on $\mathrm{p}_{+}$, even when the solutions of the previous iteration $\dot{\mathbf{u}}^{k-1}$ and $\dot{\lambda}^{k-1}$ are linear with respect to $\boldsymbol{\theta}$, the derivative $\dot{\mathbf{u}}^{k}$ may fail to have that property if the set $\left\{\lambda^{k-1}+\gamma^{k}\left(\mathbf{u}_{\mathbf{n}}^{k}-\mathbf{g}_{\mathbf{n}}\right)=0\right\}$ is not of null measure.

In light of the previous remark, we get interested in sufficient conditions for shape differentiability of $\mathbf{u}^{k}$. We introduce the corresponding subsets for problem (2.7), for each $k \geq 1$,

$$
\begin{aligned}
& \mathcal{A}^{k}:=\left\{x \in \Gamma_{C}:\left(\lambda^{k-1}+\gamma^{k}\left(\mathbf{u}_{\mathbf{n}}^{k}-\mathbf{g}_{\mathbf{n}}\right)\right)(x)>0\right\}, \\
& \mathcal{I}^{k}:=\left\{x \in \Gamma_{C}:\left(\lambda^{k-1}+\gamma^{k}\left(\mathbf{u}_{\mathbf{n}}^{k}-\mathbf{g}_{\mathbf{n}}\right)\right)(x)<0\right\}, \\
& \mathcal{B}^{k}:=\left\{x \in \Gamma_{C}:\left(\lambda^{k-1}+\gamma^{k}\left(\mathbf{u}_{\mathbf{n}}^{k}-\mathbf{g}_{\mathbf{n}}\right)\right)(x)=0\right\} .
\end{aligned}
$$

Using these notations, for any $k \geq 1$, we are able to state conditions that guarantee shape differentiability of $\mathbf{u}^{k}$, and will be referred to as Assumption 3.23 at rank $k$ :

Assumption 3.23. For each $j \in\{1, \ldots, k\}$, the set $\mathcal{B}^{j}$ is of measure zero.

Remark 3.24. This assumption is similar to Assumption 3.14, but in the case of the regularized formulation obtained at each iteration.

Corollary 3.25. Let $k \geq 1,\left(\mathbf{u}^{k}, \lambda^{k}\right)$ still denotes the solution of (2.7)-(2.8) and $\left(\dot{\mathbf{u}}^{k}, \dot{\lambda}^{k}\right)$ its material derivative, defined by (3.12)-(3.13). Under the assumptions of Theorem 3.21, if Assumption 3.23 holds at rank $k$, then the map $\boldsymbol{\theta} \mapsto\left(\dot{\mathbf{u}}^{k}, \dot{\lambda}^{k}\right)$ is linear continuous from $\boldsymbol{\Theta}$ to $\mathbf{X} \times L^{2}\left(\Gamma_{C}\right)$. Therefore, $\mathbf{u}^{k}$ is (strongly) shape differentiable in $\mathbf{L}^{2}(\Omega)$, and its shape derivative in any given direction $\boldsymbol{\theta}$ writes:

$$
d \mathbf{u}^{k}=\dot{\mathbf{u}}^{k}-\nabla \mathbf{u}^{k} \boldsymbol{\theta} .
$$

Proof. Here again, let us proceed by induction.

Base case Let $k=1$. From Assumption 3.23 at rank 1 , one gets that $\left|\mathcal{B}^{1}\right|=0$, which implies that

$$
\dot{\lambda}^{1}=R_{\mathbf{n}}^{1}\left(\mathbf{u}^{1}\right)=\chi_{\mathcal{A}^{1}}\left(\left(\lambda^{0}\right)^{\prime}+\gamma^{1}\left(\dot{\mathbf{u}}_{\mathbf{n}}^{1}-\mathbf{g}_{\mathbf{n}}^{\prime}\right)\right) \text { a.e. on } \Gamma_{C} .
$$

Therefore, $\dot{\mathbf{u}}^{1}$ is the solution of a linear variational formulation. Moreover, due to the regularities of $\lambda^{0}, \mathbf{g}_{\mathbf{n}}, \mathbf{n}$, and the expression of $L^{1}[\boldsymbol{\theta}]$, the maps

$$
\begin{aligned}
& \boldsymbol{\Theta} \ni \boldsymbol{\theta} \longmapsto\left(\lambda^{0}\right)^{\prime} \in L^{2}\left(\Gamma_{C}\right), \\
& \boldsymbol{\Theta} \ni \boldsymbol{\theta} \longmapsto \mathbf{g}_{\mathbf{n}}^{\prime} \in L^{\infty}\left(\Gamma_{C}\right) \text {, } \\
& \Theta \ni \boldsymbol{\theta} \longmapsto L^{1}[\boldsymbol{\theta}] \in \mathbf{X}^{*},
\end{aligned}
$$

are all linear continuous. Therefore, $\boldsymbol{\theta} \mapsto\left(\dot{\mathbf{u}}^{1}, \dot{\lambda}^{1}\right)$ is also linear continuous, from $\boldsymbol{\Theta}$ to $\mathbf{X} \times L^{2}\left(\Gamma_{C}\right)$. 
Inductive step Let $k \geq 1$ and assume the result of the corollary is true at iteration $k$. Now, suppose Assumption 3.23 holds at rank $k+1$. This exactly means that Assumption 3.23 holds at rank $k$ and $\left|\mathcal{B}^{k+1}\right|=0$. Thus, one has

$$
\dot{\lambda}^{k+1}=R_{\mathbf{n}}^{k+1^{\prime}}\left(\mathbf{u}^{k+1}\right)=\chi_{\mathcal{A}^{k+1}}\left(\dot{\lambda}^{k}+\gamma^{k+1}\left(\dot{\mathbf{u}}_{\mathbf{n}}^{k+1}-\mathbf{g}_{\mathbf{n}}^{\prime}\right)\right) \text { a.e. on } \Gamma_{C} .
$$

Hence, the variational formulation (3.12) is linear. Using the result at iteration $k$, one gets that $\boldsymbol{\theta} \mapsto \dot{\lambda}^{k}$ is linear continuous from $\boldsymbol{\Theta}$ to $L^{2}\left(\Gamma_{C}\right)$. This, combined with the properties of $\mathbf{g}_{\mathbf{n}}, \mathbf{n}$, and linear continuity of $L^{k+1}[\cdot]$ from $\Theta$ to $\mathbf{X}^{*}$, enables us to conclude.

\subsection{Convergence of the directional shape derivatives}

In this section, we aim at establishing the consistency of the augmented Lagrangian method with respect to shape differentiability. In other words, for any $\boldsymbol{\theta} \in \boldsymbol{\Theta}$ fixed, we get interested in convergence properties of the sequence $\left\{\left(\dot{\mathbf{u}}^{k}, \dot{\lambda}^{k}\right)\right\}_{k}$ as $k \rightarrow \infty$. Especially, we would like to derive sufficient conditions for $\left\{\dot{\mathbf{u}}^{k}\right\}_{k}$ to converge to $\dot{\mathbf{u}}$ the solution of $(3.8)$.

Remark 3.26. As we will manipulate in this section the material derivatives of $\mathbf{u}^{k}$ and $\lambda^{k}$, it will be required that Assumption 3.3 holds (see Theorem 3.9).

As for the original problem, let us define $\mathbf{w}^{k}:=\mathbf{u}^{k}-\mathbf{g} \in \mathbf{X}$. Since the existence of strong material derivatives in all directions has been established for $\mathbf{u}^{k}$ (Thm. 3.21), we get that $\mathbf{w}^{k}$ admits a strong material derivative in the direction $\boldsymbol{\theta}$ given by $\dot{\mathbf{w}}^{k}=\dot{\mathbf{u}}^{k}-\mathbf{g}^{\prime} \in \mathbf{X}$. It follows from (3.12) and (3.13) that $\dot{\mathbf{w}}^{k}$ solves:

$$
\begin{aligned}
a\left(\dot{\mathbf{w}}^{k}, \mathbf{v}\right)-L^{k}[\boldsymbol{\theta}](\mathbf{v})+a\left(\mathbf{g}^{\prime}, \mathbf{v}\right)+\left(\dot{\lambda}^{k}, \mathbf{v}_{\mathbf{n}}\right)_{\Gamma_{C}} & =0, \quad \forall \mathbf{v} \in \mathbf{X}, \\
\dot{\lambda}^{k}-d \mathbf{p}_{+}\left(\lambda^{k-1}+\gamma^{k} \mathbf{w}_{\mathbf{n}}^{k} ; \dot{\lambda}^{k-1}+\gamma^{k} \dot{\mathbf{w}}_{\mathbf{n}}^{k}\right) & =0, \quad \text { a.e. on } \Gamma_{C} .
\end{aligned}
$$

Note that $(3.14 \mathrm{~b})$ can be equivalently rewritten as:

$$
\dot{\lambda}^{k}= \begin{cases}\dot{\lambda}^{k-1}+\gamma^{k} \dot{\mathbf{w}}_{\mathbf{n}}^{k} & \text { on } \mathcal{A}^{k}, \\ 0 & \text { on } \mathcal{I}^{k} \\ \mathrm{p}_{+}\left(\dot{\lambda}^{k-1}+\gamma^{k} \dot{\mathbf{w}}_{\mathbf{n}}^{k}\right) & \text { on } \mathcal{B}^{k}\end{cases}
$$

In order to have a valid expression for all $k \geq 1$, the notation $\dot{\lambda}^{0}:=\lambda^{0^{\prime}}$ will be used. Finally, we give some straightforward but very useful properties of $d \mathrm{p}_{+}$:

- for all $u, v \in \mathbb{R}$, one has $d \mathrm{p}_{+}(u ; v) v=\left|d \mathrm{p}_{+}(u ; v)\right|^{2}$,

- $d \mathrm{p}_{+}$is continuous on $\mathbb{R}^{2} \backslash(\{0\} \times \mathbb{R})$.

Theorem 3.27. For any increasing sequence of strictly positive parameters $\left\{\gamma^{k}\right\}_{k}$, there exists a subsequence of $\left\{\left(\dot{\mathbf{w}}^{k}, \dot{\lambda}^{k}\right)\right\}_{k}$ that is bounded in $\mathbf{X} \times H^{-\frac{1}{2}}\left(\Gamma_{C}\right)$.

Proof. For now, let $\left\{\gamma^{k}\right\}_{k} \subset \mathbb{R}_{+}^{*}$ be any increasing sequence, from which the ALM algorithm generates iterates $\left(\mathbf{u}^{k}, \lambda^{k}\right)$. From Theorem 2.11, one gets that $\left\{\left(\mathbf{u}^{k}, \lambda^{k}\right)\right\}_{k}$ is bounded in $\mathbf{X} \times L^{2}\left(\Gamma_{C}\right)$, from which one deduces that $\left\{L^{k}[\boldsymbol{\theta}]\right\}_{k}$ is bounded in $\mathbf{X}^{*}$. Thus, taking $\mathbf{v}=\dot{\mathbf{w}}^{k}$ as test-function in (3.14a) yields

$$
\alpha_{0}\left\|\dot{\mathbf{w}}^{k}\right\|_{\mathbf{X}}^{2}+\left(\dot{\lambda}^{k}, \dot{\mathbf{w}}_{\mathbf{n}}^{k}\right)_{\Gamma_{C}} \leq C\left\|\dot{\mathbf{w}}^{k}\right\|_{\mathbf{X}} .
$$


Now, we rewrite the second term, then use the properties of $d \mathrm{p}_{+}$and Young's inequality:

$$
\begin{aligned}
\left(\dot{\lambda}^{k}, \dot{\mathbf{w}}_{\mathbf{n}}^{k}\right)_{\Gamma_{C}} & =\frac{1}{\gamma^{k}}\left(\dot{\lambda}^{k}, \dot{\lambda}^{k-1}+\gamma^{k} \dot{\mathbf{w}}_{\mathbf{n}}^{k}\right)_{\Gamma_{C}}-\frac{1}{\gamma^{k}}\left(\dot{\lambda}^{k}, \dot{\lambda}^{k-1}\right)_{\Gamma_{C}} \\
& =\frac{1}{\gamma^{k}}\left\|\dot{\lambda}^{k}\right\|_{0, \Gamma_{C}}^{2}-\frac{1}{\gamma^{k}}\left(\dot{\lambda}^{k}, \dot{\lambda}^{k-1}\right)_{\Gamma_{C}} \\
& \geq \frac{1}{2 \gamma^{k}}\left(\left\|\dot{\lambda}^{k}\right\|_{0, \Gamma_{C}}^{2}-\left\|\dot{\lambda}^{k-1}\right\|_{0, \Gamma_{C}}^{2}\right) .
\end{aligned}
$$

Plugging this into inequality (3.16) leads to

$$
\alpha_{0}\left\|\dot{\mathbf{w}}^{k}\right\|_{\mathbf{X}}^{2}-C\left\|\dot{\mathbf{w}}^{k}\right\|_{\mathbf{X}}+\frac{1}{2 \gamma^{k}}\left\|\dot{\lambda}^{k}\right\|_{0, \Gamma_{C}}^{2} \leq \frac{1}{2 \gamma^{k}}\left\|\dot{\lambda}^{k-1}\right\|_{0, \Gamma_{C}}^{2} \leq \frac{1}{2 \gamma^{k-1}}\left\|\dot{\lambda}^{k-1}\right\|_{0, \Gamma_{C}}^{2},
$$

since $\gamma^{k} \geq \gamma^{k-1}$. From here, let us distinguish the two possible cases.

(i) Case $\left\{\frac{1}{\gamma^{k}}\left\|\dot{\lambda}^{k}\right\|_{0, \Gamma_{C}}^{2}\right\}_{k}$ bounded.

Boundedness of the whole sequence $\left\{\dot{\mathbf{w}}^{k}\right\}_{k}$ in $\mathbf{X}$ follows directly from (3.18).

(ii) Case $\left\{\frac{1}{\gamma^{k}}\left\|\dot{\lambda}^{k}\right\|_{0, \Gamma_{C}}^{2}\right\}_{k}$ unbounded.

In that case, let us sum (3.18) from 1 to $k$, with $k>1$ :

$$
\sum_{l=1}^{k}\left(\alpha_{0}\left\|\dot{\mathbf{w}}^{l}\right\|_{\mathbf{X}}^{2}-C\left\|\dot{\mathbf{w}}^{l}\right\|_{\mathbf{X}}\right)+\frac{1}{2 \gamma^{k}}\left\|\dot{\lambda}^{k}\right\|_{0, \Gamma_{C}}^{2} \leq \frac{1}{2 \gamma^{1}}\left\|\dot{\lambda}^{0}\right\|_{0, \Gamma_{C}}^{2} .
$$

If we denote $W^{k}$ the first term of the left hand side, it follows that the sequence $\left\{W^{k}\right\}_{k}$ is unbounded below. Thus there exists a subsequence (denoted $\varphi(k))$ such that $\left\{W^{\varphi(k)}\right\}_{k}$ is strictly monotone and tends to $-\infty$. Especially, strict monotonicity of this subsequence implies that, for all $k \geq 1$,

$$
W^{\varphi(k+1)}-W^{\varphi(k)}=\sum_{l=\varphi(k)}^{\varphi(k+1)}\left(\alpha_{0}\left\|\dot{\mathbf{w}}^{l}\right\|_{\mathbf{X}}^{2}-C\left\|\dot{\mathbf{w}}^{l}\right\|_{\mathbf{X}}\right)<0
$$

Hence, for all $k \geq 1$, there exists $\psi(k) \in \llbracket \varphi(k), \varphi(k+1) \rrbracket$ such that $\alpha_{0}\left\|\dot{\mathbf{w}}^{\psi(k)}\right\|_{\mathbf{X}}^{2}-C\left\|\dot{\mathbf{w}}^{\psi(k)}\right\|_{\mathbf{X}}<0$, which proves that $\left\{\dot{\mathbf{w}}^{\psi(k)}\right\}_{k}$ is bounded in $\mathbf{X}$.

Then, boundedness of $\left\{\dot{\lambda}^{k}\right\}_{k}$ or of a subsequence of $\left\{\dot{\lambda}^{k}\right\}_{k}$ in $H^{-\frac{1}{2}}\left(\Gamma_{C}\right)$ immediately follows from (3.14a) and the subjectivity of the trace operator from $\mathbf{X}$ to $\mathbf{H}^{\frac{1}{2}}\left(\Gamma_{C}\right)$.

Corollary 3.28. There exists an increasing sequence of strictly positive parameters $\left\{\gamma^{k}\right\}_{k}$ such that the whole sequence $\left\{\left(\dot{\mathbf{w}}^{k}, \dot{\lambda}^{k}\right)\right\}_{k}$ defined by (3.14) is bounded in $\mathbf{X} \times H^{-\frac{1}{2}}\left(\Gamma_{C}\right)$. 
Proof. From estimation (3.18), it is clear that if the sequence of parameters $\left\{\gamma^{k}\right\}_{k}$ is built from some $\gamma^{1}>0$ following the rule, for $k>1$,

$$
\gamma^{k}=\max \left\{\gamma^{k-1},\left\|\dot{\lambda}^{k-1}\right\|_{0, \Gamma_{C}}^{2}\right\}
$$

then one automatically gets boundedness of the whole sequence $\left\{\dot{\mathbf{w}}^{k}\right\}_{k}$ in $\mathbf{X}$. In other words, such a choice of parameters ensures that we are in case (i) of the previous proof. Again, boundedness of $\left\{\dot{\lambda}^{k}\right\}_{k}$ in $H^{-\frac{1}{2}}\left(\Gamma_{C}\right)$ follows.

Of course, the previous result implies that for any sequence of parameters, the associated iterates $\left(\dot{\mathbf{w}}^{k}, \dot{\lambda}^{k}\right)$ converge weakly in $\mathbf{X} \times H^{-\frac{1}{2}}\left(\Gamma_{C}\right)$ to some limit $(\hat{\mathbf{w}}, \hat{\lambda})$, up to a subsequence. In order to specify this limit, we need to make the following additional assumption.

Assumption 3.29. The iterates $\lambda^{k}$ generated by the ALM converge to $\lambda$ a.e. on $\Gamma_{C}$.

Remark 3.30. Because of the non-continuity of $d \mathrm{p}_{+}$on $\{0\} \times \mathbb{R}$ getting a convergence result without Assumption 3.14 seems rather difficult (maybe even impossible).

Lemma 3.31. Let $\left\{\gamma^{k}\right\}_{k}$ be any increasing sequence in $\mathbb{R}_{+}^{*},\left(\mathbf{u}^{k}, \lambda^{k}\right)$ still denotes the solution of (2.7)-(2.8), and $(\mathbf{u}, \lambda)$ the solution of (2.4). When Assumptions 3.14 and 3.29 hold, the characteristic functions $\chi_{\mathcal{A}^{k}}$ (respectively $\chi_{\mathcal{B}^{k}}$ ) converge to $\chi_{\mathcal{A}}$ (respectively 0 ) strongly in $L^{p}\left(\Gamma_{C}\right)$, for each $1<p<+\infty$, up to a subsequence.

Proof. First of all, note that since $\mathcal{A}^{k}$ and $\mathcal{A}$ are measurable, so are $\chi_{\mathcal{A}^{k}}$ and $\chi_{\mathcal{A}}$, for any $k \geq 0$. Since those functions are also bounded on $\Gamma_{C}$ which is of finite measure, they both belong to $L^{1}\left(\Gamma_{C}\right)$, and thus to any $L^{p}\left(\Gamma_{C}\right)$ with $1<p<+\infty$. Now, let us begin with proving pointwise convergence, from which we will deduce weak then strong convergence.

Since $\mathbf{u}^{k} \rightarrow \mathbf{u}$ strongly in $L^{2}\left(\Gamma_{C}\right)$, there exists a subsequence, still denoted $\mathbf{u}^{k}$, that converges a.e. on $\Gamma_{C}$. From now, we consider this subsequence. Then, from Assumption 3.14, for a.e. $x \in \Gamma_{C}, x$ is either in $\mathcal{A}$, or in $\mathcal{I}$.

- If $x \in \mathcal{A}$, then there is some $\delta>0$ such that $\lambda(x)>\delta$. From Assumption 3.29, one gets that $\exists k_{0}>0, \forall k \geq k_{0}$, $\left|\lambda^{k}(x)-\lambda(x)\right|<\delta / 2$. Especially, for all $k \geq k_{0}$,

$$
\lambda^{k+1}(x)=\mathrm{p}_{+}\left(\lambda^{k}(x)+\gamma^{k+1}\left(\mathbf{u}_{\mathbf{n}}^{k+1}(x)-\mathbf{g}_{\mathbf{n}}(x)\right)\right)>\delta / 2 .
$$

Thus, for all $k \geq k_{0}+1, \chi_{\mathcal{A}^{k}}(x)=1=\chi_{\mathcal{A}}(x)$, and $\chi_{\mathcal{B}^{k}}(x)=0$.

- If $x \in \mathcal{I}$, then $\lambda(x)=0$ and there is some $\delta^{\prime}>0$ such that $\mathbf{u}_{\mathbf{n}}(x)-\mathbf{g}_{\mathbf{n}}(x)<-\delta^{\prime} / \gamma^{1}$. Moreover, $\exists k_{0}^{\prime}>0$ such that $\forall k \geq k_{0}^{\prime},\left|\mathbf{u}_{\mathbf{n}}^{k}(x)-\mathbf{u}_{\mathbf{n}}(x)\right|<\delta^{\prime} / 2 \gamma^{1}$ and $\lambda^{k}(x)<\delta^{\prime} / 4$. Consequently, for such values of $k$, one has

$$
\lambda^{k}(x)+\gamma^{k+1}\left(\mathbf{u}_{\mathbf{n}}^{k+1}(x)-\mathbf{g}_{\mathbf{n}}(x)\right)<\delta^{\prime} / 4-\gamma^{k+1} \delta^{\prime} / 2 \gamma^{1} \leq-\delta^{\prime} / 4 .
$$

Hence, for any $k \geq k_{0}^{\prime}+1$, one gets $\chi_{\mathcal{A}^{k}}(x)=0=\chi_{\mathcal{A}}(x)$, and $\chi_{\mathcal{B}^{k}}(x)=0$.

This proves that $\chi_{\mathcal{A}^{k}} \rightarrow \chi_{\mathcal{A}}$ and $\chi_{\mathcal{B}^{k}} \rightarrow 0$ a.e. on $\Gamma_{C}$. Let $1<p<+\infty$. For $\left\{\chi_{\mathcal{B}^{k}}\right\}_{k}$, strong convergence follows directly from Lebesgue's dominated convergence theorem and the fact that $\left|\chi_{\mathcal{B}^{k}}\right|^{p} \leq 1$ a.e. on $\Gamma_{C}$. For $\left\{\chi_{\mathcal{A}^{k}}\right\}_{k}$, since the sequence is bounded in $L^{p}\left(\Gamma_{C}\right)$, weak convergence in $L^{p}\left(\Gamma_{C}\right)$ follows. Taking for example $p=2$, one gets that

$$
\int_{\Gamma_{C}} \chi_{\mathcal{A}^{k}}=\int_{\Gamma_{C}} \chi_{\mathcal{A}^{k}} \cdot 1 \longrightarrow \int_{\Gamma_{C}} \chi_{\mathcal{A}}
$$


Obviously, this also proves that $\left\|\chi_{\mathcal{A}^{k}}\right\|_{L^{p}\left(\Gamma_{C}\right)} \rightarrow\left\|\chi_{\mathcal{A}}\right\|_{L^{p}\left(\Gamma_{C}\right)}$. For such values of $p, L^{p}\left(\Gamma_{C}\right)$ is uniformly convex, therefore weak convergence and convergence of the norms imply strong convergence.

Theorem 3.32. Suppose Assumptions 3.14 and 3.29 hold. Then, choosing the parameters $\gamma^{k}$ as in Corollary 3.28 leads to one of the two following cases:

(i) $\left\{\gamma^{k}\right\}_{k}$ is bounded, then the whole sequence $\left\{\dot{\mathbf{w}}^{k}\right\}_{k}$ converges to $\dot{\mathbf{w}}$, the solution of (3.10), strongly in $\mathbf{X}$,

(ii) $\left\{\gamma^{k}\right\}_{k}$ is unbounded, then $\left\{\dot{\mathbf{w}}^{k}\right\}_{k}$ converges weakly to some limit $\hat{\mathbf{w}} \in \mathbf{X}_{\mathcal{A}}$, up to a subsequence.

Proof. As mentioned above, a direct consequence of Theorem 3.27 is that, up to a subsequence, the iterates $\left(\dot{\mathbf{w}}^{k}, \dot{\lambda}^{k}\right)$ converge weakly to some limit $(\hat{\mathbf{w}}, \hat{\lambda}) \in \mathbf{X} \times H^{-\frac{1}{2}}\left(\Gamma_{C}\right)$. In the following, let us consider that subsequence, which we still denote using the superscript ${ }^{k}$. Due to strong convergence $\left(\mathbf{u}^{k}, \lambda^{k}\right) \rightarrow(\mathbf{u}, \lambda)$ in $\mathbf{X} \times H^{-\frac{1}{2}}\left(\Gamma_{C}\right)$, it is clear that $L^{k}[\boldsymbol{\theta}] \rightarrow L[\boldsymbol{\theta}]$ strongly in $\mathbf{X}^{*}$, where $L[\boldsymbol{\theta}]$ is defined as: for all $\mathbf{v} \in \mathbf{X}$,

$$
L[\boldsymbol{\theta}](\mathbf{v}):=L^{\prime}(\mathbf{v})-a^{\prime}(\mathbf{u}, \mathbf{v})-\int_{\Gamma_{C}} \lambda \mathbf{v}_{\mathbf{n}} \operatorname{div}_{\Gamma} \boldsymbol{\theta}
$$

Consequently, the weak limit satisfies:

$$
a(\hat{\mathbf{w}}, \mathbf{v})-L[\boldsymbol{\theta}](\mathbf{v})+a\left(\mathbf{g}^{\prime}, \mathbf{v}\right)+\left\langle\hat{\lambda}, \mathbf{v}_{\mathbf{n}}\right\rangle_{\Gamma_{C}}=0, \quad \forall \mathbf{v} \in \mathbf{X}
$$

From now, we distinguish two possible cases.

(i) Case $\left\{\gamma^{k}\right\}_{k}$ bounded.

From the definition of $\left\{\gamma^{k}\right\}_{k}$ (see (3.19)), it follows that $\left\{\dot{\lambda}^{k}\right\}_{k}$ is bounded in $L^{2}\left(\Gamma_{C}\right)$. Therefore, there exists a subsequence that converges weakly in $L^{2}\left(\Gamma_{C}\right)$ to $\hat{\lambda}$ (due to uniqueness of the weak limit), which also proves that $\hat{\lambda} \in L^{2}\left(\Gamma_{C}\right)$. As $\left\{\gamma^{k}\right\}_{k}$ is an increasing bounded sequence, it converges, say to $\hat{\gamma}>0$. Now, let $\eta \in L^{4}\left(\Gamma_{C}\right)$, one has:

$$
\begin{aligned}
\left(\dot{\lambda}^{k}, \eta\right)_{\Gamma_{C}} & =\left(\dot{\lambda}^{k-1}+\gamma^{k} \dot{\mathbf{w}}_{\mathbf{n}}^{k}, \eta\right)_{\mathcal{A}^{k}}+\left(\dot{\lambda}^{k}, \eta\right)_{\mathcal{B}^{k}} \\
& =\left(\dot{\lambda}^{k-1}, \chi_{\mathcal{A}^{k}} \eta\right)_{\Gamma_{C}}+\gamma^{k}\left(\dot{\mathbf{w}}_{\mathbf{n}}^{k}, \chi_{\mathcal{A}^{k}} \eta\right)_{\Gamma_{C}}+\left(\dot{\lambda}^{k}, \chi_{\mathcal{B}^{k}} \eta\right)_{\Gamma_{C}} .
\end{aligned}
$$

We know that $\dot{\lambda}^{k}, \dot{\mathbf{w}}_{\mathbf{n}}^{k}$ converge weakly in $L^{2}\left(\Gamma_{C}\right)$, and using the results of Lemma 3.31 with $p=4$, it follows that $\chi_{\mathcal{A}^{k}} \eta, \chi_{\mathcal{B}^{k}} \eta$ converge strongly in $L^{2}\left(\Gamma_{C}\right)$. Thus, passing to the limit on both sides of the equality yields:

$$
\hat{\lambda}=\chi_{\mathcal{A}}\left(\hat{\lambda}+\hat{\gamma} \hat{\mathbf{w}}_{\mathbf{n}}\right) \text { in } L^{\frac{4}{3}}\left(\Gamma_{C}\right)
$$

From this, one deduces that $\hat{\mathbf{w}} \in \mathbf{X}_{\mathcal{A}}$ and

$$
a(\hat{\mathbf{w}}, \mathbf{v})-L^{\prime}(\mathbf{v})+a^{\prime}(\mathbf{u}, \mathbf{v})+a\left(\mathbf{g}^{\prime}, \mathbf{v}\right)=0, \quad \forall \mathbf{v} \in \mathbf{X}_{\mathcal{A}}
$$

which means that $\hat{\mathbf{w}}=\dot{\mathbf{w}}$, since the solution of this problem is unique. Uniqueness also proves that the whole sequence converges weakly to $\dot{\mathbf{w}}$. 
Finally, to get strong convergence, let us take $\mathbf{v}=\dot{\mathbf{w}}^{k}$ as test-function in (3.14a). Since the embedding $H^{\frac{1}{2}}\left(\Gamma_{C}\right) \hookrightarrow L^{2}\left(\Gamma_{C}\right)$ is compact, one obtains for the second term of $(3.14 \mathrm{a})$ :

$$
\left(\dot{\lambda}^{k}, \dot{\mathbf{w}}_{\mathbf{n}}^{k}\right)_{\Gamma_{C}} \longrightarrow\left(\hat{\lambda}, \dot{\mathbf{w}}_{\mathbf{n}}\right)_{\Gamma_{C}}=0
$$

from which we get that $a\left(\dot{\mathbf{w}}^{k}, \dot{\mathbf{w}}^{k}\right) \rightarrow a(\dot{\mathbf{w}}, \dot{\mathbf{w}})$. The ellipticity of $a$ finishes the proof.

(ii) Case $\left\{\gamma^{k}\right\}_{k}$ unbounded.

Again, let us take $\mathbf{v}=\dot{\mathbf{w}}^{k}$ as test-function in (3.14a), and rewrite the second term of the formulation.

$$
\begin{aligned}
\left(\dot{\lambda}^{k}, \dot{\mathbf{w}}_{\mathbf{n}}^{k}\right)_{\Gamma_{C}} & =\left(\dot{\lambda}^{k-1}+\gamma^{k} \dot{\mathbf{w}}_{\mathbf{n}}^{k}, \dot{\mathbf{w}}_{\mathbf{n}}^{k}\right)_{\mathcal{A}^{k}}+\left(\dot{\lambda}^{k}, \dot{\mathbf{w}}_{\mathbf{n}}^{k}\right)_{\mathcal{B}^{k}} \\
& \geq \gamma^{k}\left\|\dot{\mathbf{w}}_{\mathbf{n}}^{k}\right\|_{0, \mathcal{A}^{k}}^{2}-C\left(\left\|\dot{\lambda}^{k-1}\right\|_{-1 / 2, \Gamma_{C}}+\left\|\dot{\lambda}^{k}\right\|_{-1 / 2, \Gamma_{C}}\right)\left\|\dot{\mathbf{w}}^{k}\right\|_{\mathbf{X}} .
\end{aligned}
$$

Using this estimation in (3.14a), one obtains that $\left\{\gamma^{k}\left\|\dot{\mathbf{w}}_{\mathbf{n}}^{k}\right\|_{0, \mathcal{A}^{k}}^{2}\right\}_{k}$ is bounded. Using Lemma 3.31 with $p=2$ and the continuous embedding $H^{\frac{1}{2}}\left(\Gamma_{C}\right) \hookrightarrow L^{4}\left(\Gamma_{C}\right)$, one obtains, up to a subsequence,

$$
\left\|\dot{\mathbf{w}}_{\mathbf{n}}^{k}\right\|_{0, \mathcal{A}^{k}}^{2}=\int_{\Gamma_{C}} \chi_{\mathcal{A}^{k}} \cdot\left|\dot{\mathbf{w}}_{\mathbf{n}}^{k}\right|^{2} \longrightarrow\left\|\hat{\mathbf{w}}_{\mathbf{n}}\right\|_{0, \mathcal{A}}^{2} .
$$

On the other hand, since $\left\{\gamma^{k}\right\}_{k}$ is unbounded, there exists a subsequence (of the previous subsequence) that diverges to $+\infty$. Hence, boundedness of $\left\{\gamma^{k}\left\|\dot{\mathbf{w}}_{\mathbf{n}}^{k}\right\|_{0, \mathcal{A}^{k}}^{2}\right\}_{k}$ implies that $\left\|\hat{\mathbf{w}}_{\mathbf{n}}\right\|_{0, \mathcal{A}}^{2}=0$, that is $\hat{\mathbf{w}} \in \mathbf{X}_{\mathcal{A}}$.

Remark 3.33. The previous result relies on a specific choice of parameters $\left\{\gamma^{k}\right\}_{k}$, defined by (3.19), that may not be easy to build in practice. Moreover, Assumption 3.29 is essential in the proof of Lemma 3.31, and thus of Theorem 3.32. However it seems difficult to give an interpretation of Assumption 3.29 and, although Theorem 2.11 ensures weak convergence of $\left\{\lambda^{k}\right\}_{k}$ in $L^{2}\left(\Gamma_{C}\right)$, convergence almost everywhere seems to be a strong assumption. For those reasons, this (partial) convergence result should be understood as follows: there exist cases where we are able to prove consistency of the ALM with respect to shape sensitivity analysis.

\subsection{Shape derivative of a generic criterion}

As we aim at expressing first order optimality conditions related to problem (3.2), we need to find an expression for the shape derivative of the functional $J^{k}$. From now, let us consider functionals which take the generic form:

$$
J^{k}(\Omega)=\mathcal{J}\left(\Omega, \mathbf{u}^{k}(\Omega)\right):=\int_{\Omega} l\left(\mathbf{u}^{k}(\Omega)\right)+\int_{\partial \Omega} m\left(\mathbf{u}^{k}(\Omega)\right)
$$

Let us make the usual regularity assumptions: the functions $l, m$ are $\mathcal{C}^{1}\left(\mathbb{R}^{d}, \mathbb{R}\right)$, and their derivatives, denoted $l^{\prime}, m^{\prime}$, are Lipschitz. It is also assumed that those functions and their derivatives satisfy, for all $u, v \in \mathbb{R}^{d}$,

$$
\begin{aligned}
|l(u)| \leq C\left(1+|u|^{2}\right), & |m(u)| \leq C\left(1+|u|^{2}\right), \\
\left|l^{\prime}(u) \cdot v\right| \leq C|u \cdot v|, & \left|m^{\prime}(u) \cdot v\right| \leq C|u \cdot v|,
\end{aligned}
$$


for some constants $C>0$. Let us state the well known shape differentiability result for such functionals $J^{k}$ (see, e.g. $[16])$.

Theorem 3.34. Let $k \geq 1$. Under the assumptions of Theorem 3.21, $J^{k}$ is conically shape differentiable at $\Omega$, and its derivative in the direction $\boldsymbol{\theta} \in \mathbf{\Theta}$ writes:

$$
d J^{k}(\Omega)[\boldsymbol{\theta}]=\int_{\Omega}\left(l^{\prime}\left(\mathbf{u}^{k}\right) \cdot \dot{\mathbf{u}}^{k}+l\left(\mathbf{u}^{k}\right) \operatorname{div} \boldsymbol{\theta}\right)+\int_{\partial \Omega}\left(m^{\prime}\left(\mathbf{u}^{k}\right) \cdot \dot{\mathbf{u}}^{k}+m\left(\mathbf{u}^{k}\right) \operatorname{div}_{\Gamma} \boldsymbol{\theta}\right) .
$$

Corollary 3.35. Let $k \geq 1$. In addition to the previous result, when the assumptions of Corollary 3.25 are verified, the map $\boldsymbol{\theta} \mapsto d J^{k}(\Omega)[\boldsymbol{\theta}]$ is linear from $\boldsymbol{\Theta}$ to $\mathbb{R}$. Thus, $J^{k}$ is classically shape differentiable at $\Omega$.

Remark 3.36. It follows from Theorem 3.27 that $d J^{k}$ is bounded for suitable choices of parameters $\gamma^{k}$. Therefore, formula (3.23) produces usable shape derivatives, regardless how many iterations of the ALM algorithm are performed. Moreover, a sufficient condition to get convergence of $\left\{d J^{k}\right\}_{k}$ is that $\left\{\dot{\mathbf{u}}^{k}\right\}_{k}$ converges weakly in $\mathbf{X}$.

\section{Conclusion}

In this work, we have expressed sufficient conditions for shape differentiability of $\mathbf{u}$, the solution to the original Signorini problem. We also got interested in shape differentiability properties for the sequence of the iterates $\mathbf{u}^{k}$ (approaching $\mathbf{u}$ ) obtained when applying the augmented Lagrangian method to this problem. After proving that these iterates were always conically shape differentiable, we have given sufficient conditions for the associated shape derivatives to converge to the shape derivative of $\mathbf{u}$. On the other hand, we also found conditions that guarantee shape differentiability of the iterates.

A natural extension of the present work would be to consider the contact problem with Tresca friction. For this model, proving conical shape differentiabilty of $\mathbf{u}^{k}$ and expressing sufficient conditions for $\mathbf{u}^{k}$ to be shape differentiable is rather straightforward since the arguments from [3] apply. However, proving conical shape differentiability and deriving sufficient conditions for classical shape differentiability of the solution to the associated variational inequality seems more difficult. Indeed, for this problem, conical shape differentiability has been proved only in the two-dimensional case and for specific directions $\boldsymbol{\theta}$, see [33].

\section{REFERENCES}

[1] P. Beremlijski, J. Haslinger, M. Kočvara and J. Outrata, Shape optimization in contact problems with Coulomb friction. SIAM J. Optim. 13 (2002) 561-587.

[2] P. Beremlijski, J. Haslinger, J. Outrata and R. Pathó, Shape optimization in contact problems with Coulomb friction and a solution-dependent friction coefficient. SIAM J. Control Optim. 52 (2014) 3371-3400.

[3] B. Chaudet-Dumas and J. Deteix, Shape derivatives for the penalty formulation of elastic contact problems with Tresca friction. SIAM J. Control Optim. 58 (2020) 3237-3261.

[4] P.G. Ciarlet (Ed.), Mathematical Elasticity, Vol. I: Three-Dimensional Elasticity. Studies in Mathematics and Its Applications. Academic Press, Elsevier (1988) 20.

[5] M.C. Delfour and J.-P. Zolésio, A boundary differential equation for thin shells. J. Differ. Eq. 119 (1995) $426-449$.

[6] M.C. Delfour and J.-P. Zolésio, Shapes and Geometries: Analysis, Differential Calculus, and Optimisation. Society for Industrial and Applied Mathematics, Philadelphia, PA (2001).

[7] J. Deny, Théorie de la capacité dans les espaces fonctionnels. Sémin. Brelot-Choquet-Deny. Théor. potentiel 9 (1965) 1-13.

[8] C. Eck, J. Jarusek and M. Krbec, Unilateral Contact Problems: Variational Methods and Existence Theorems. CRC Press (2005).

[9] I. Ekeland and R. Temam, Convex Analysis and Variational Problems, Vol. 28. Siam (1999).

[10] M. Fortin and R. Glowinski, Augmented Lagrangian Methods: Applications to the Numerical Solution of Boundary-Value Problems. Studies in Mathematics and Its Applications. Elsevier Science (2000).

[11] J. Haslinger and A. Klarbring, Shape optimization in unilateral contact problems using generalized reciprocal energy as objective functional. Nonlinear Anal.: Theory Methods Appl. 21 (1993) 815-834.

[12] J. Haslinger and P. Neittaanmäki, Finite Element Approximation for Optimal Shape Design: Theory and Applications. John Wiley \& Sons (1988). 
[13] J. Haslinger and P. Neittaanmäki, Finite Element Approximation for Optimal Shape, Material, and Topology Design. John Wiley \& Sons (1996).

[14] J. Haslinger, J. Outrata and R. Pathó, Shape optimization in 2D contact problems with given friction and a solution-dependent coefficient of friction. Set-Valued Var. Anal. 20 (2012) 31-59.

[15] C. Heinemann and K. Sturm, Shape optimization for a class of semilinear variational inequalities with applications to damage models. SIAM J. Math. Anal. 48 (2016) 3579-3617.

[16] A. Henrot and M. Pierre, Shape Variation and Optimization: A Geometrical Analysis. European Mathematical Society Publishing House (2018).

[17] M. Hintermüller and A. Laurain, Optimal shape design subject to elliptic variational inequalities. SIAM J. Control Optim. 49 (2011) 1015-1047.

[18] K. Ito and K. Kunisch, Lagrange Multiplier Approach to Variational Problems and Applications. SIAM (2008).

[19] J. Jarušek, M. Krbec, M. Rao and J. Sokołowski, Conical differentiability for evolution variational inequalities. J. Differ. Eq. 193 (2003) 131-146.

[20] N. Kikuchi and J.T. Oden, Contact Problems in Elasticity: A Study of Variational Inequalities and Finite Element Methods, Vol. 8. SIAM (1988).

[21] A. Klarbring and J. Haslinger, On almost constant contact stress distributions by shape optimization. Struct. Optim. 5 (1993) $213-216$.

[22] M. Kočvara and J. Outrata, On optimization of systems governed by implicit complementarity problems. Numer. Funct. Anal. Optim. 15 (1994) 869-887.

[23] A. Maury, Shape Optimization for Contact and Plasticity Problems Thanks to the Level Set Method. Ph.D. thesis, Université Pierre et Marie Curie-Paris VI (2016).

[24] F. Mignot, Contrôle dans les inéquations variationelles elliptiques. J. Funct. Anal. 22 (1976) 130-185.

[25] F. Mignot and J.-P. Puel, Optimal control in some variational inequalities. SIAM J. Control Optim. 22 (1984) $466-476$.

[26] F. Murat and J. Simon, Étude de problèmes d'optimal design, in IFIP Technical Conference on Optimization Techniques. Springer (1975), 54-62.

[27] P. Neittaanmäki, J. Sokolowski and J.-P. Zolésio. Optimization of the domain in elliptic variational inequalities. Appl. Math. Optim. 18 (1988) 85-98.

[28] J. Sokolowski and J.-P. Zolésio, Shape sensitivity analysis for variational inequalities, in System Modeling and Optimization. Springer (1982), 401-407.

[29] J. Sokolowski and J.-P. Zolésio, Dérivée par rapport au domaine de la solution d'un problème unilatéral. C. R. Acad. Sc. Paris 301 (1985) 103-106.

[30] J. Sokolowski and J.-P. Zolésio, Sensitivity analysis of elastic-plastic torsion problem, in System Modelling and Optimization. Springer (1986) 845-853.

[31] J. Sokolowski and J.-P. Zolésio, Shape design sensitivity analysis of plates and plane elastic solids under unilateral constraints. J. Optim. Theory Appl. 54 (1987) 361-382.

[32] J. Sokolowski and J.-P. Zolésio, Shape sensitivity analysis of unilateral problems. SIAM J. Math. Anal. 18 (1987) $1416-1437$.

[33] J. Sokołowski and J.-P. Zolésio, Shape sensitivity analysis of contact problem with prescribed friction. Nonlinear Anal.: Theory Methods Appl. 12 (1988) 1399-1411.

[34] J. Sokolowski and J.-P. Zolésio, Introduction to shape optimization, in Introduction to Shape Optimization. Springer (1992).

[35] J. Sokolowski and J.-P. Zolésio, Differential stability of solutions to unilateral problems. Free Bound. Probl.: Appl. Theory 4 (1993) 537-547.

[36] G. Stadler, Infinite-dimensional semi-smooth Newton and augmented Lagrangian methods for friction and contact problems in elasticity. Selbstverl. (2004).

[37] L.M. Susu. Optimal control of a viscous two-field gradient damage model. GAMM-Mitteilungen 40 (2018) $287-311$. 